\title{
Small-molecule fluorescence-based probes for aging diagnosis
}

\author{
Donglei Shia ${ }^{a, b}, 1$, Wenwen Liu ${ }^{c, 1}$, Guangwei Wang ${ }^{b}$, Yuan Guo ${ }^{b, *}$ and Jian $\operatorname{Li}^{a, c, d, e, *}$ \\ aState Key Laboratory of Bioreactor Engineering, Shanghai Frontiers Science Center of Optogenetic Techniques for Cell Metabolism, Frontiers \\ Science Center for Materiobiology and Dynamic Chemistry, Shanghai Key Laboratory of New Drug Design, School of Pharmacy, East China \\ University of Science and Technology, Shanghai 200237, China \\ ${ }^{b}$ College of Chemistry and Materials Science, Northwest University, Xi'an 710127, China \\ cKey Laboratory of Tropical Biological Resources of Ministry of Education, College of Pharmacy, Hainan University, Haikou 570228, Hainan, \\ China \\ dYunnan Key Laboratory of Screening and Research on Anti-Pathogenic Plant Resources from West Yunnan, College of Pharmacy, Dali \\ University, Dali 671000, China \\ eClinical Medicine Scientific and Technical Innovation Center, Shanghai Tenth People's Hospital, Tongji University School of Medicine, \\ Shanghai 200092, China \\ ${ }^{1}$ These authors contributed equally to this work: Donglei Shi, Wenwen Liu. \\ *Correspondence: jianli@ecust.edu.cn (J. Li); guoyuan@nwu.edu.cn (Y. Guo)
}

Received: 03 December 2021; Revised: 18 December 2021; Accepted: 20 December 2021

Published online: 12 January 2022

DOI 10.15212/AMM-2021-0004

\begin{abstract}
Aging is a time-dependent decline in physiological function that affects most organisms and is the major risk factor for many non-communicable diseases. The early diagnosis of aging is critical for the treatment of aging and agingrelated diseases. In recent years, extensive efforts have attempted to accurately diagnose aging. To date, multiple types of fluorescent probes for various age-related biomarkers have been developed, with the aims of achieving rapid, precise diagnosis of the aging process and evaluating the efficacy of anti-aging drugs. This review summarizes recent research progress in small-molecule fluorescence-based probes for aging diagnosis, and further discusses the challenges and opportunities in this field.
\end{abstract}

Keywords: fluorescent probes, aging, senescence, diagnosis for aging, age-related biomarkers

\section{INTRODUCTION}

Aging is a time-dependent decline in physiological integrity that leads to physical function, a high risk of chronic diseases and vulnerability to death [1]. Several aging pathways and processes, such as the insulin-like signaling pathway, oxidative stress, chronic inflammation, mitochondrial dysfunction and cellular senescence, are associated with a myriad of age-related diseases including type 2 diabetes, neurodegenerative diseases, cardiovascular disease and cancer frailty [2,3]. During the past 40 years, the demand for anti-aging medicine has shifted from sick care after occurring of diseases to preventive healthcare applications, and the risk factors for disease development are more concerning before disease onset [3]. As such, early identification and interventions for aging and age-related diseases are the basis for "healthy aging" in the general population. Aging research has achieved great success in elucidating the genetic pathways that underlie aging phenotypes, and the number of candidate anti-aging drugs discovered in model organisms has substantially grown [4]. However, no anti-aging drugs have currently been approved, largely because of a lack of reliable drug targets and effective methods for evaluating anti-aging efficacy. Thus, in the field of aging research, an urgent need exists to develop effective methods to monitor the aging process, assess the efficacy of interventions and predict individuals' healthy lifespans.

With the development of aging research, many aging-related biomarkers originating from multiple signaling pathways have emerged [5]. Typically, the activity of senescence-associated $\beta$-galactosidase (SA- $\beta$-gal) enhances in senescent cells as the number of lysosomes increases [6]. The persistent DNA-damage response induces the phosphorylation of histone H2AX and p53; therefore, $\gamma \mathrm{H} 2 \mathrm{AX}$ nuclear foci and phosphorylated p53 are commonly used as age-related biomarkers [7, 8]. Cyclin-dependent-kinase inhibitors, including p16, p15 and p21, are the main drivers of the cell-cycle arrest in cellular senescence [9-11]. Additionally, senescent cells secrete senescence-associated 
secretory phenotype (SASP) proteins, including IL-1A, IL-1B, IL-6, IL-8, and MMP-1, which contribute to dysfunction in several biological processes [12]. On the basis of those age-related biomarkers, common methods for the diagnosis of aging include SA- $\beta$-gal staining by $\mathrm{X}$-gal, and the detection of proteins and mRNAs by western blotting and $\mathrm{qPCR}$, respectively. However, these methods are complicated, time-consuming and particularly inappropriate for in vivo biological imaging. Because of the advantages of time savings, high sensitivity, flexible molecular design strategies and imageable features, various fluorescent probes for age-related biomarkers have been developed for the diagnosis of aging.

Herein, we review recent advancements and insights in the development of small-molecule fluorescence-based probes for the diagnosis of aging (Table 1). These probes were constructed on the basis of different biomarkers, including small active molecules, microenvironmental parameters and various enzyme types. Furthermore, several probes based on unique detection strategies for aging have been designed. We hope that this review will help researchers design more precise and valuable molecular tools for the diagnosis of aging.

\section{DIAGNOSIS OF AGING}

\subsection{Fluorescent probes for aging diagnosis based on enzymes and other markers}

Sialic acid (SIA) is a type of anionic monosaccharide, which is usually located at the termini of glycoconjugates

Table 1 | Summary of optical properties and biological applications of small-molecule fluorescence-based probes for aging diagnosis

\begin{tabular}{|c|c|c|c|c|c|}
\hline Probe & $\lambda_{\mathrm{ex}} / \lambda_{\mathrm{em}}(\mathrm{nm})$ & Detection approach & Imaging applications & Biomarkers & Reference \\
\hline Sia-RQ & $580 / 610$ & Turn-on & Cells & Sialidases & {$[14]$} \\
\hline TP-SS & $\begin{array}{l}435 / 570 \\
550 / 675\end{array}$ & Ratiometric & Cells, mice & GPx & [17] \\
\hline MitoCy- $\mathrm{NH}_{2}$ & $\begin{array}{l}730 / 770-810 \\
(650 / 700-740)\end{array}$ & Ratiometric & Cells, mice & MAO-B & [19] \\
\hline QM-NHofuc & $543 / 586$ & Turn-on & Cells, mice & $\alpha$-L-fucosidase & {$[22]$} \\
\hline NIR-BG2 & $675 / 709$ & Turn-on & Cells, mice & $\beta$-gal & {$[25]$} \\
\hline BOD-L- $\beta$ Gal & $\begin{array}{l}488 / 574 \\
(533 / 727)\end{array}$ & Ratiometric & Cells, mice & $\beta$-gal & {$[26]$} \\
\hline YDGAL & $500 / 585$ & Turn-on & $\begin{array}{l}\text { Cells, tissues and } \\
\text { organs of mice }\end{array}$ & $\beta$-gal & {$[27]$} \\
\hline HeckGal & $488 / 550$ & Turn-on & $\begin{array}{l}\text { Cells, tumors and } \\
\text { kidneys of mice }\end{array}$ & $\beta$-gal & [28] \\
\hline KSL11 & $425 / 662$ & Turn-on & Cells & $\beta$-gal & [31] \\
\hline KSL0401 & $435 / 706$ & Turn-on & Cells & $\beta$-gal & [32] \\
\hline TR-G & $550 / 590(638)$ & Ratiometric & Cells, mice & $\beta$-gal & [33] \\
\hline Comp. 1 & $488 / 624$ & Turn-on & Cells & $\beta$-gal & [34] \\
\hline SRP & $495 / 545$ & Turn-on & Cells & $\beta$-gal & [35] \\
\hline $1 a$ & $365 / 510$ & Ratiometric & Cells & $\mathrm{H}_{2} \mathrm{O}_{2}$ & [38] \\
\hline QX-B & $725 / 772$ & Turn-on & Cells, mice & $\mathrm{H}_{2} \mathrm{O}_{2}$ & [39] \\
\hline Mito-Bor & $700 / 730$ & Turn-on & Cells, mice & $\mathrm{H}_{2} \mathrm{O}_{2}$ & [40] \\
\hline KSLP1 & $560 / 700$ & Light-up & Cells, C. elegans & Polarity & {$[44]$} \\
\hline SGJ & $380 / 480$ & Light-up & Cells & $\mathrm{pH}$ & {$[47,48]$} \\
\hline СуВС9 & $649 / 670$ & Light-up & Cells & Membrane potential & [49] \\
\hline KSA01 & $460 / 536$ & \multirow[t]{2}{*}{ Ratiometric } & \multirow[t]{2}{*}{ Cells, tissues of mice } & \multirow{2}{*}{$\begin{array}{l}\text { Two-dimensional: } \\
\mathrm{pH}, \beta \text {-gal }\end{array}$} & \multirow[t]{2}{*}[50]{} \\
\hline KSA02 & $460 / 534$ & & & & \\
\hline ROKS & $\begin{array}{l}350 / 534 \\
350 / 534(602)\end{array}$ & Turn-on; Ratiometric & Cells, C. elegans & $\begin{array}{l}\text { Stress-response } \\
\text { capability }\end{array}$ & [51] \\
\hline
\end{tabular}


on the surfaces of mammalian cells. The terminal SIA residues can be hydrolyzed by sialidase. Desialylation mediates a variety of biological processes, such as pathogenicity and cell inflammation [13]. To investigate the relationship between desialylation and cellular senescence, Han et al. have developed a fluorescent probe (Sia-RQ) for detecting sialidases [14]. Sia-RQ is composed of the very bright fluorophore rhodamine- $X$ and a self-immolative moiety of 4-hydroxymandelic acid combined with a SIA as the recognition group for sialidase (Figure 1). After desialylation, Sia-RQ undergoes self-immolation and releases a fluorescent, reactive receptor (quinone methide-rhodamine- $X$ dyad), which further binds sialidases. SDS-PAGE and in-gel fluorescence imaging have indicated that Sia-RQ can covalently label sialidase after enzymatic activation. More importantly, Sia-RQ has been successfully used to assess the expression level of sialidase in senescent Huh7 cells incubated with palbociclib for 0-14 days. The red-channel fluorescence of senescent cells dramatically increased at $3 \mathrm{~d}$ and plateaued at 7-14 d after palbociclib stimulation, thus indicating that the expression of sialidase was upregulated with senescence.

Glutathione peroxidase (GPx), a selenocysteine (Sec)containing protein, is a crucial protease in the front-line antioxidant defense system, which is involved in the regulation of redox homeostasis and the pentose phosphate pathway [15]. Oxidative stress has been demonstrated to become more severe during the aging process. GPx, a central factor in the GPx/GSH redox pool, plays a crucial role in this process [16]. Chen et al. have reported the two-photon fluorescent probe TP-SS for the ratiometric detection of GPx [17], which consists of the two-photon chromophore TP-NH and a 2,2'-dithiodiethanol (response site) (Figure 2A). TP-SS exhibits a rapid response, high selectivity and stability for the detection of Sec which serves as the active site for GPx in vitro. The probe has been used to monitor the fluctuation of GPx in senescent cells and the catalytic cycle of the GPx/GSH redox pool in live cells (Figure 2B). Thus, this probe can be used to monitor the catalytic cycle of the GPx/GSH redox pool and evaluate oxidative stress in living cells. Importantly, using TP-SS, the authors have monitored changes in GPx in mice at different ages (Figure 2C) and observed a decrease in GPx activity with aging.

Monoamine oxidase (MAO) is a flavoenzyme that is located in the outer membrane of mitochondria and catalyzes the oxidative deamination of neurotransmitters, thus producing aldehydes and hydrogen peroxide. MAOs have two isoforms: MAO-A and MAO-B. The latter generates reactive oxygen species (ROS), which cause the oxidation of biogenic amines to the corresponding aldehydes [18]. MAO-B levels are higher in young than old individuals, and consequently are a potential biomarker for senescence detection. Chen et al. have reported two probes (MitoCy- $\mathrm{NH}_{2}$ and MitoHCy- $\mathrm{NH}_{2}$ ) to synergistically monitor MAO-B and its contribution to oxidative stress [19]. Both probes consist of heptamethine cyanine, propanamide and a triphenylphosphonium cation. As shown in Figure 3A, MitoCy- $\mathrm{NH}_{2}$ displays ratiometric fluorescence responses toward MAO-B. In contrast, MitoHCy- $\mathrm{NH}_{2}$ enables logic-based detection of $\mathrm{O}_{2}{ }^{--}$(a by-product of oxidation of biogenic amines catalyzed by MAO-B) and MAO-B. MitoHCy- $\mathrm{NH}_{2}$ has been used to monitor the activity of MAO-B and ROS $\left(\mathrm{O}_{2}{ }^{-}\right)$ in replicative-senescent HepG2. The level of MAO-B

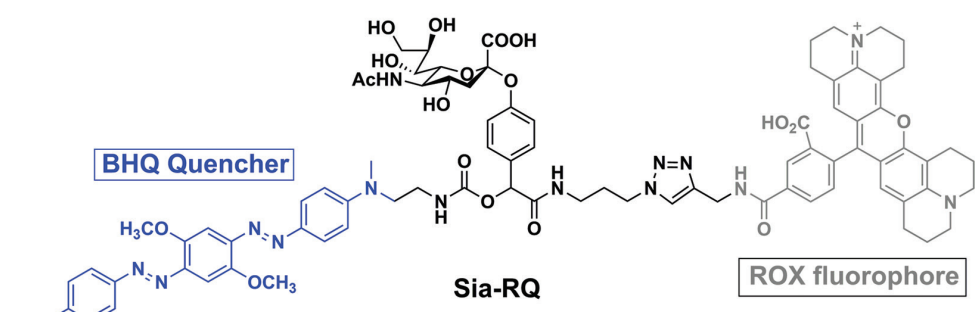

Fluorescence-quenching
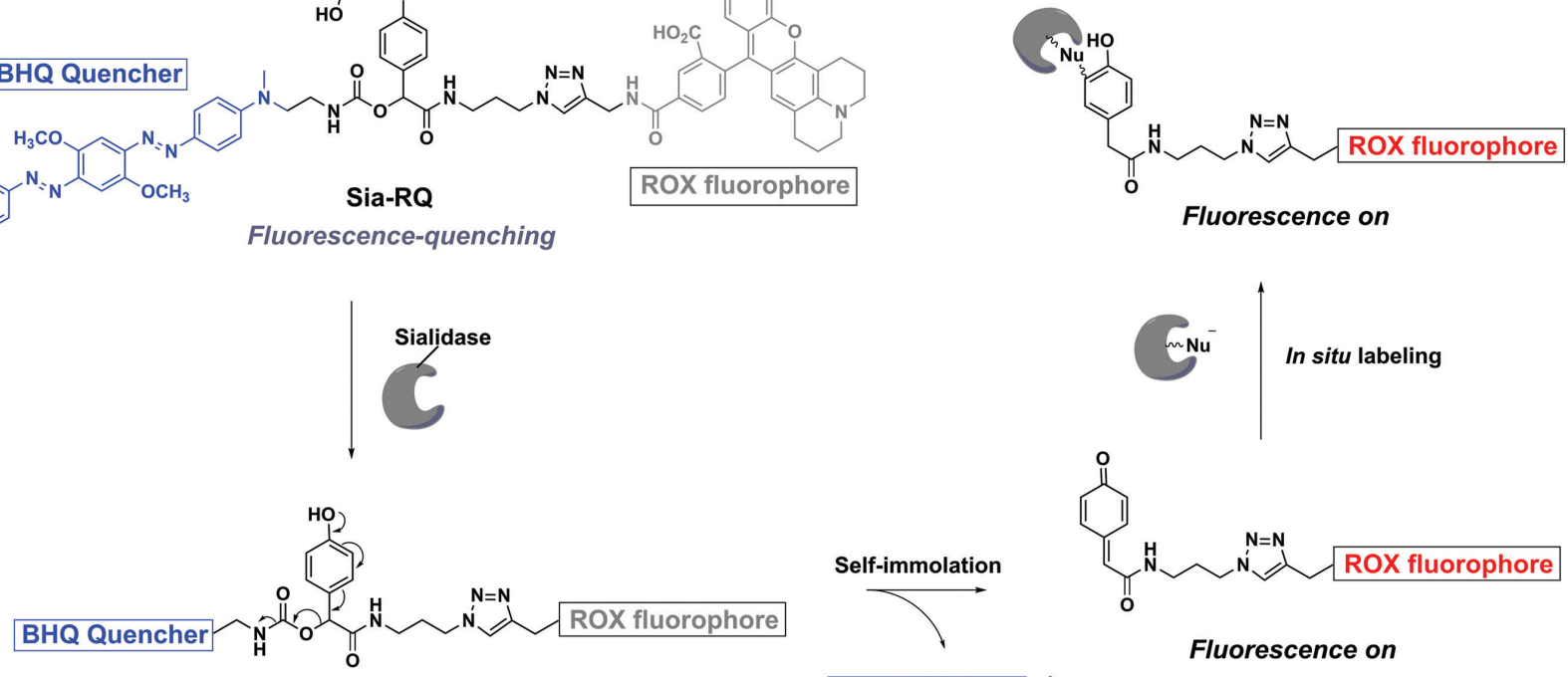

Fluorescence on

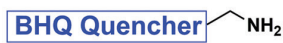

Figure 1 | The chemical structure of Sia-RQ and the proposed detection mechanism. 
A<smiles>CC[N+]1=C(/C=C/c2ccc3cc(N(C)C(=O)OCCSSCCO)ccc3c2)C(C)(C)c2ccccc21</smiles>
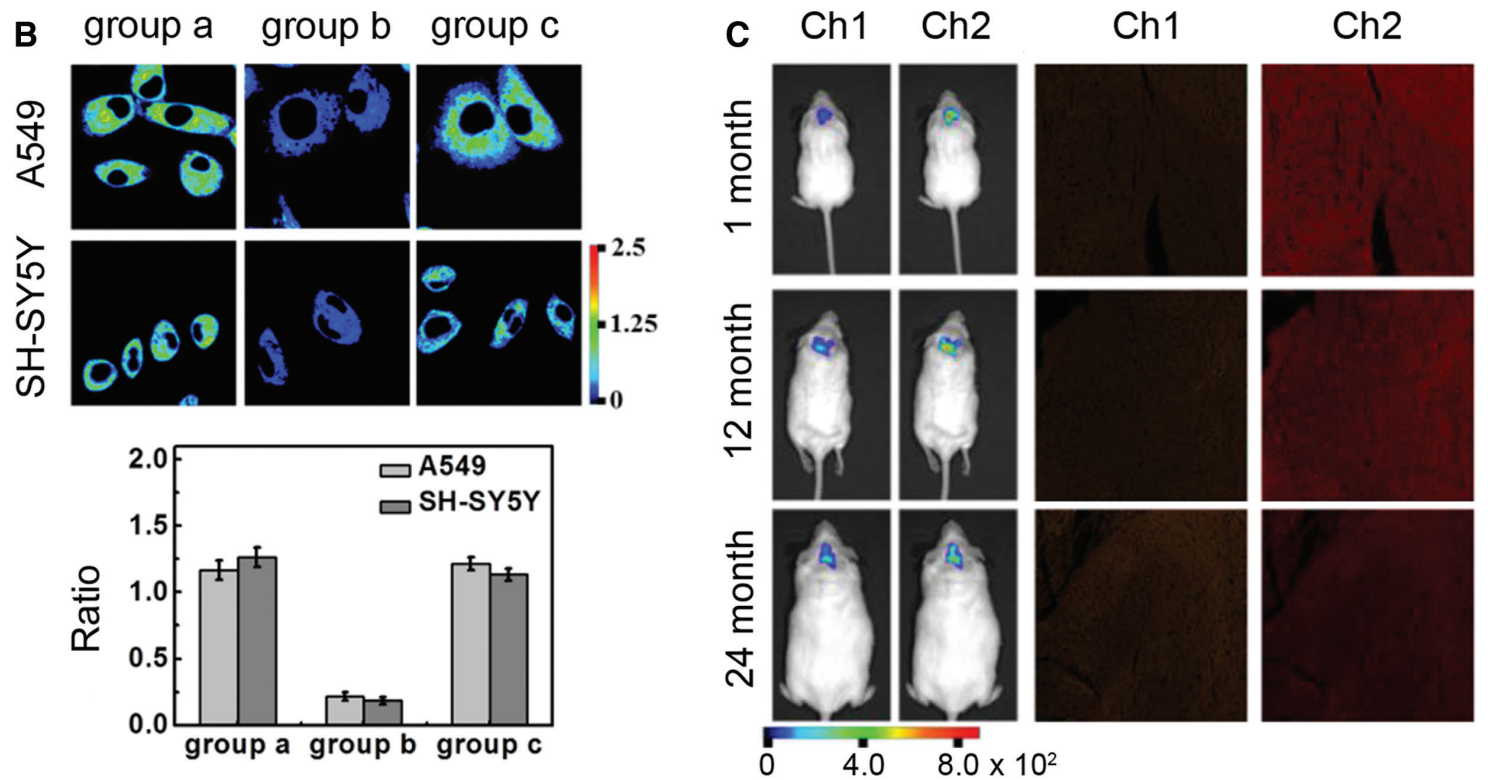

Figure 2 | (A) The chemical structure of TP-SS. (B) TP-SS fluorescence imaging of GPx in living cells. Cells in group a were incubated with $10 \mu \mathrm{M}$ TP-SS: cells in group b were incubated with $10 \mu \mathrm{M} \mathrm{Hg}{ }^{2+}$ and $10 \mu \mathrm{M}$ TP-SS; cells in group c were incubated $10 \mu \mathrm{M} \mathrm{Hg}{ }^{2+}, 10 \mu \mathrm{M} \mathrm{Sec}$ and $10 \mu \mathrm{M}$ TP-SS. The fluorescence intensity ratio of TP-SS-loaded cells in different groups is shown. (C) TP-SS fluorescence images of GPX in aged mice and in fresh brain slices. Adapted with permission from Ref. [17]. Copyright 2021 American Chemical Society.

increases with senescence, and the generated ROS may exacerbate apoptosis (Figure 3B). In addition, the probe MitoCy- $\mathrm{NH}_{2}$ has successfully enabled selective identification of MAO-B in $\mathrm{H}_{2} \mathrm{O}_{2}$-induced senescent cells and in aged mice in a ratiometric near-infrared (NIR) fluorescence response (Figure 3B,C).

$\alpha$-Fucosidase ( $\alpha$-Fuc) is a lysosomal glycosidase encoded by FUCA1, which is involved in the decomposition of glycoproteins, oligosaccharides and glycolipids. In all canonical types of cellular senescence (replicative, oncogene-induced and DNA-damage-induced senescence), the mRNA expression levels and enzyme activity of $\alpha$-Fuc are up-regulated; consequently, this enzyme is an emerging biomarker for senescence $[20,21]$. Kim et al. have reported the aggregation-induced emission (AIE) probe QM-NHofuc for detecting $\alpha$-Fuc (Figure 4A). The probe comprises a quinolone-malononitrile scaffold and AIEgen core and a hydrophilic $\alpha$-fucopyranoside group as the response site of $\alpha$-Fuc [22]. The $\alpha$-Fuc expression in several cellular senescence models has been evaluated with chromogenic X-fuc assays in multiple types of senescent cells overexpressing $\alpha$-Fuc. The ability of QM-NH $\alpha$ fuc to detect $\alpha$-Fuc has been verified in those senescent cell types (Figure 4B). Moreover, the ability of QM-NHofuc to track aging has been evaluated in drug-induced aged xenograft-tumor mice (Figure 4C).

SA- $\beta$-gal, a lysosomal enzyme encoded by the GLB1 gene, is widely used as a biomarker to track senescence [23]. The activity of SA- $\beta$-gal is directly associated with cellular senescence and has been regarded as the "gold standard" for monitoring senescence [24]. Recently, Cui et al. constructed the self-immobilizing NIR fluorogenic probe NIR-BG2 [25]. A difluoromethyl group was incorporated in the probe, so that the electrophilic quinone methide species can be released and trapped by surrounding nucleophilic proteins after activation by SA- $\beta$-gal, thus retaining NIR signals (Figure 5). NIR-BG2 produces a marked fluorescence enhancement at 709 $\mathrm{nm}$ after reaction with SA- $\beta$-gal. The results of fluorescent western blot assays have shown that the NIR fluorescence signal absolutely overlaps with the green fluorescence signal, thus indicating that NIR-BG2 can be activated and further labeled with $\beta$-galactosidasde ( $\beta$-gal). NIR-BG2 can be used to monitor endogenous 
A

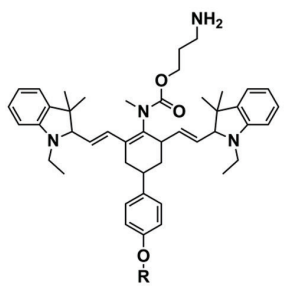

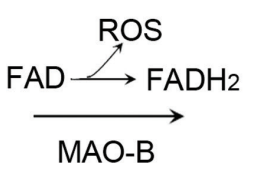

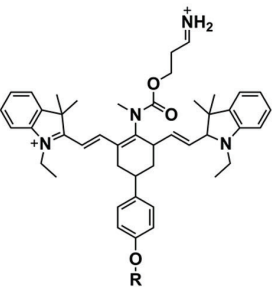

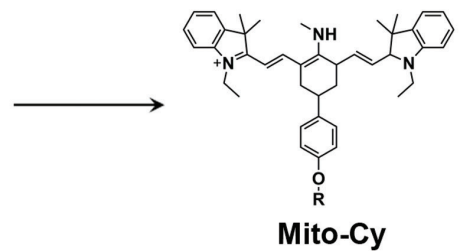

MitoHCy-NH

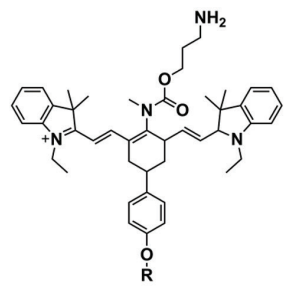

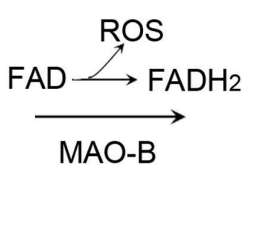

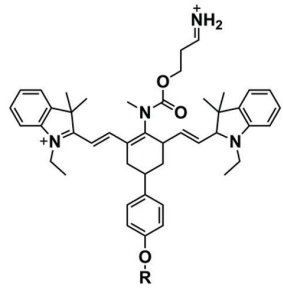

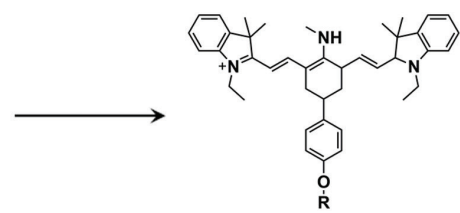

MitoCy-NH

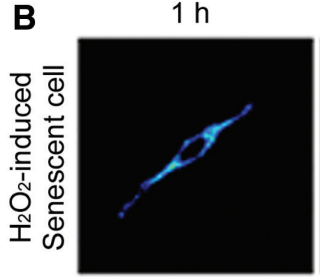

CPD 3

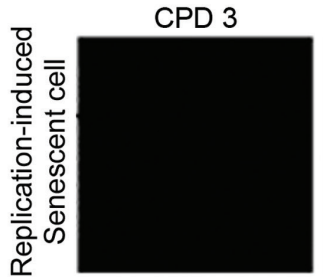

$2 \mathrm{~h}$

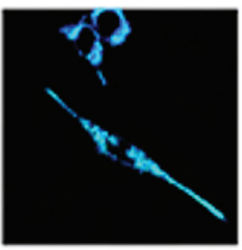

CPD 15

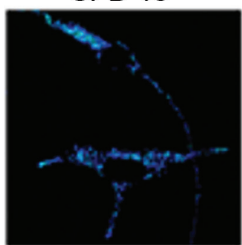

$3 \mathrm{~h}$

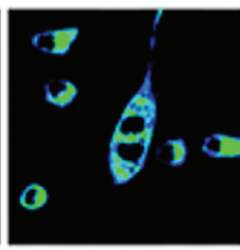

CPD 30

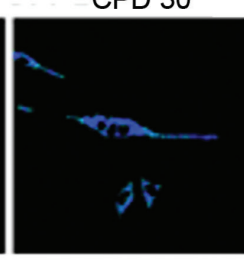

$4 \mathrm{~h}$

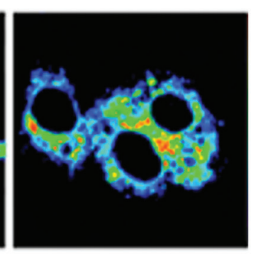

CPD 50
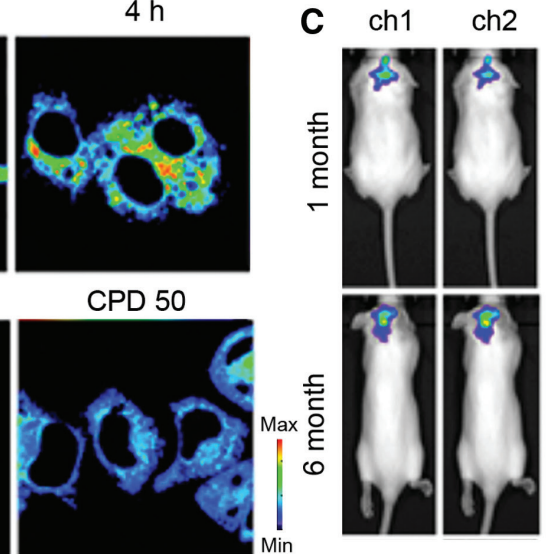

$R=N_{N}^{N=N} \sim N_{\mathrm{PPh}_{3}}^{+}$
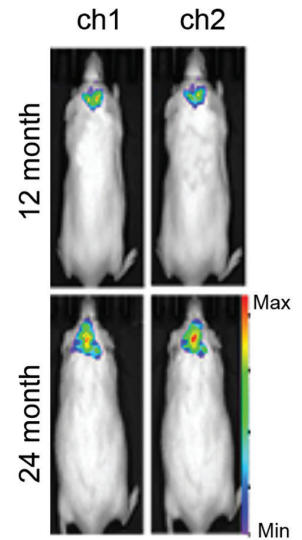

Figure 3 | (A) Chemical structures and the sensing mechanism of both probes. (B) Synergistic imaging of MAO-B and $\mathrm{O}_{2}{ }^{--}$in senescent cells. (C) MitoCy- $\mathbf{N H}_{2}$ fluorescence imaging of brains and various organs in mice at different ages. Adapted with permission from Ref. [19]. Copyright 2021 American Chemical Society.

$\beta$-gal in CT26.CL25 cells and SA- $\beta$-gal in camptothecin-induced senescent Hela cells. Notably, in vivo fluorescence imaging experiments have indicated that NIR-BG2 is suitable for the long-term tracking of SA- $\beta$-gal activity in vivo, owing to the prolonged signal retention.

$\mathrm{Gu}$ et al. have developed the nanoprobe BOD-L- $\beta$ GalNPs for the detection of $\beta$-gal. The authors first designed the NIR ratiometric probe BOD-L- $\beta$ Gal [26] by introducing a $\beta$-D-galactosyl group into boron dipyrromethene fluorophore (BODIPY) via a self-elimination linker (Figure 6A). BOD-L- $\beta$ Gal enabled the real-time detection of $\beta$-gal in senescent cells (Figure $6 \mathrm{~B}$ ). However, because BOD-L- $\beta$ Gal exhibited poor cellular uptake, the authors incorporated the probe into polymeric nanoparticles consisting of poly (lactic-co-glycolic) acid to construct the nano-probe BOD-L- $\beta$ Gal-NPs. With BOD-L- $\beta$ Gal-NPs, senescent cells and the vascular system of atherosclerotic mice have successfully been visualized (Figure 6C). Therefore, the probe may have great potential in the early diagnosis of atherosclerosis and other age-related diseases.

Lin et al. have developed the two-photon fluorescent probe YDGAL for monitoring the activity of $\beta$-gal [27]. The probe has good affinity $\left(K_{m}=12.35 \mu \mathrm{M}\right)$, a rapid response (stable within $10 \mathrm{~min}$ ), high selectivity and an extremely low limit of detection $\left(2.185 \times 10^{-6} \mathrm{U} / \mathrm{mL}\right)$ (Figure 7A,B). YDGAL has also been used to evaluate the anti-aging therapeutic effects of a combination of dasatinib (D) and quercetin (Q). As shown in Figure 7C, the cells in the control group showed weak fluorescence and exhibited strong red fluorescence because of the high SA- $\beta$-gal expression. The red-channel fluorescence of senescent cells markedly decreased after treatment with anti-aging drugs $(D+Q)$. In addition, the probe can be used to track aging process and assess the efficacy of anti-aging drugs at the organ level.

Ramón et al. have presented the new two-photon naphthalimide-styrene probe HeckGal, which 


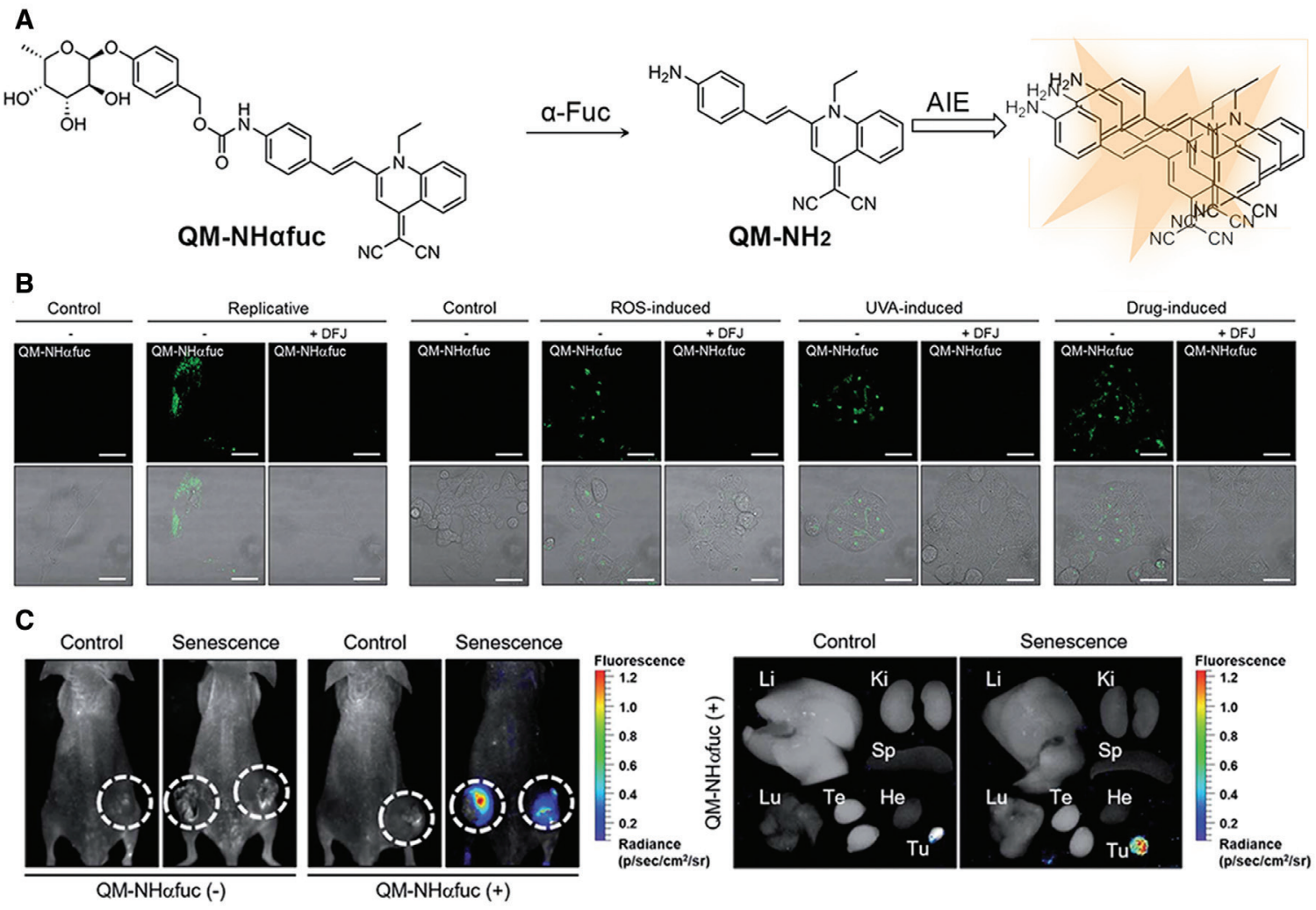

Figure 4 | (A) The chemical structure and detection mechanism of QM-NHofuc. (B) Fluorescence imaging of the probe in different types of senescent cells. (C) QM-NH $\alpha$ fuc fluorescence imaging of senescent cells in mice. Scale bar: 50 um. Adapted with permission from Ref. [22]. Copyright 2021 The Royal Society of Chemistry.

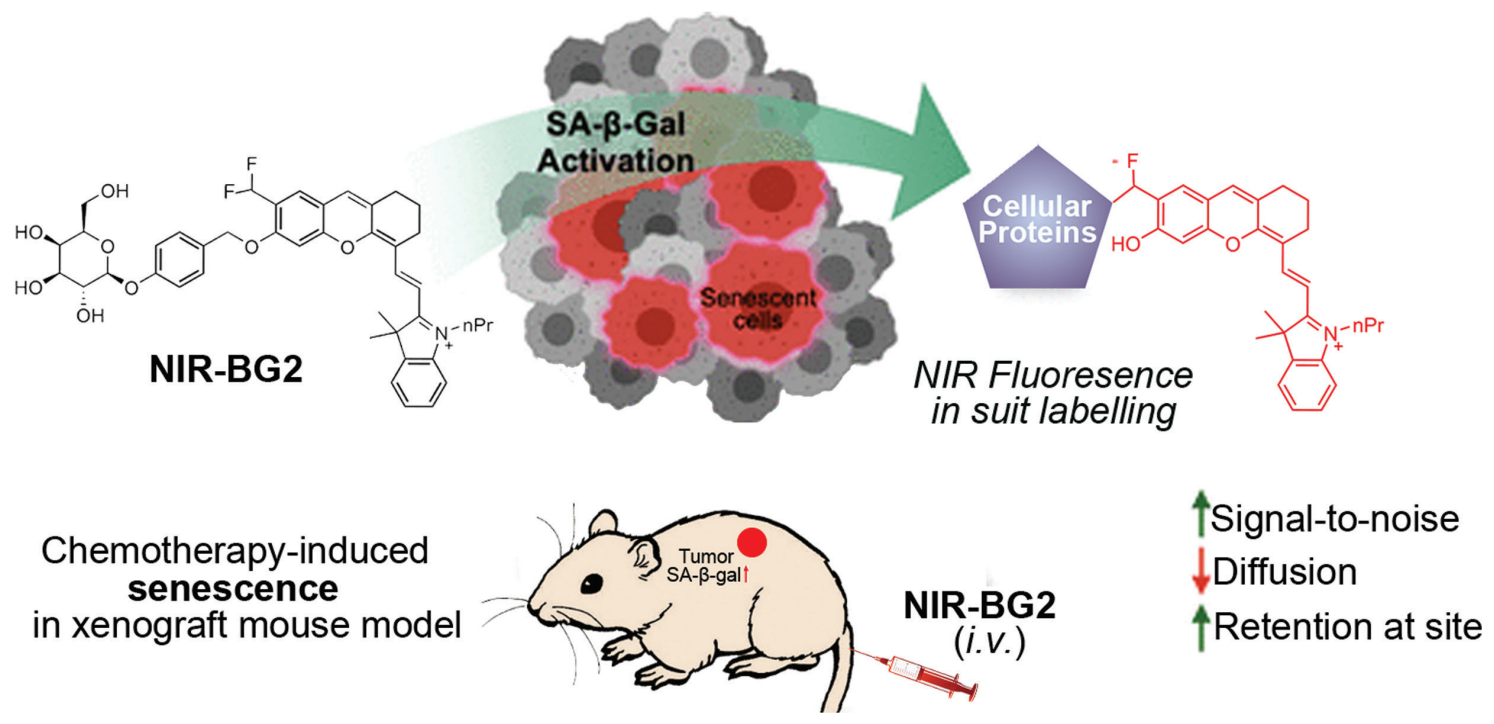

Figure 5 | Chemical structure of the probe NIR-BG2 and schematic diagram of the detection of senescent cells in vivo by using this probe. Adapted with permission from Ref. [25]. Copyright 2021 American Chemical Society.

consists of a fluorophore (naphthalimide-stryene) and an acetylated $\beta$-galactose (Figure 8A) [28]. HeckGal has poor emission performance and a low fluorescence quantum yield of 0.074 . However, after hydrolysis with $\beta$-gal, the detection system exhibits an 11.8-fold enhancement in fluorescence quantum yield $(0.8754)$. 

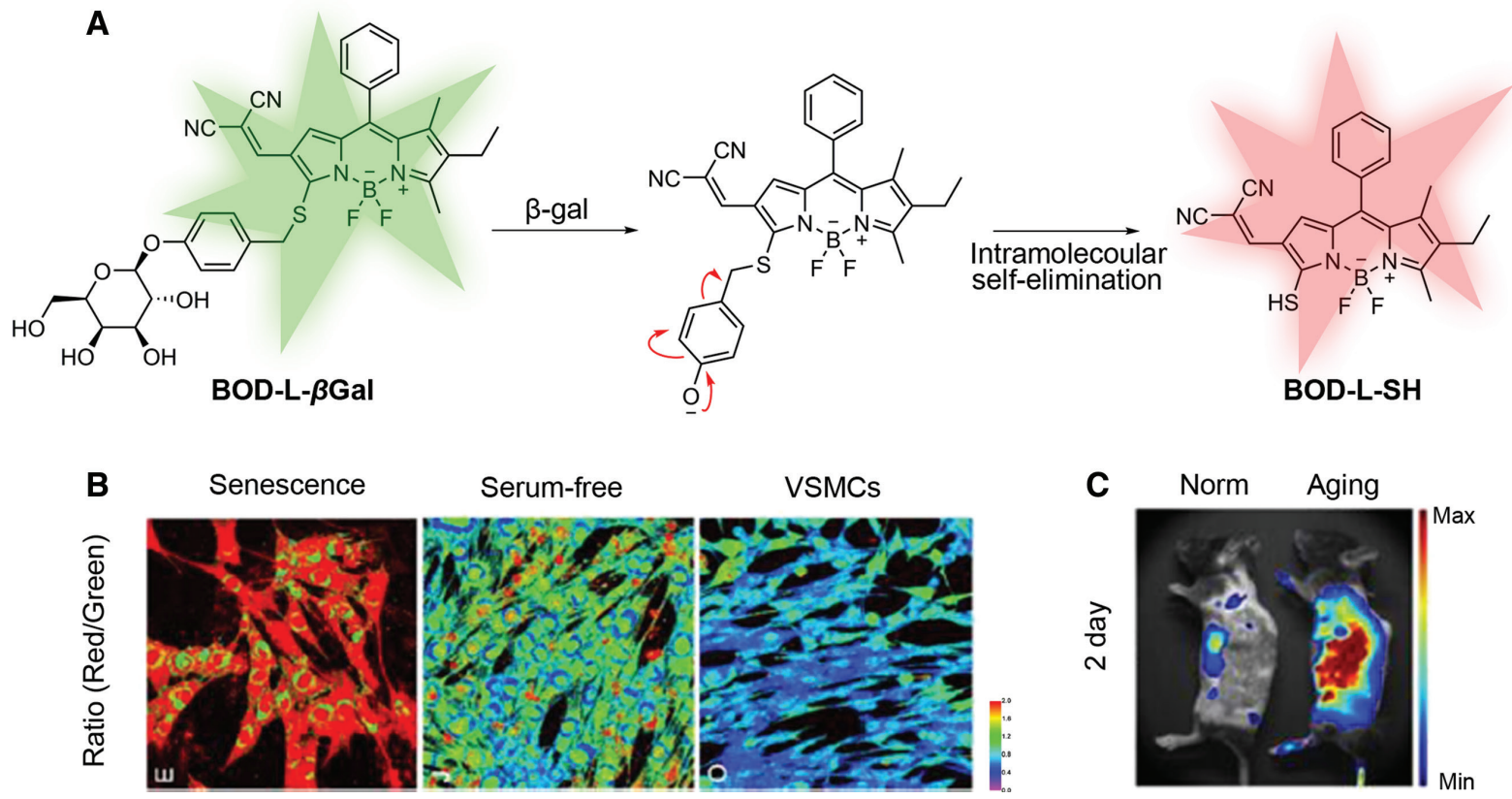

Figure 6 | (A) Molecular design of the fluorescent probe BOD-L- $\beta$ Gal and its sensing mechanism. (B) Ratiometric images of VSMCS including Ang II-induced senescent VSMCs, serum-free-treated VSMCs and primary VSMCs. (C) BOD-L- $\beta$ Gal-NPs fluorescence imaging of atherosclerotic mice and control mice. (B) and (C) Adapted with permission from Ref. [26]. Copyright 2021 American Chemical Society.

A

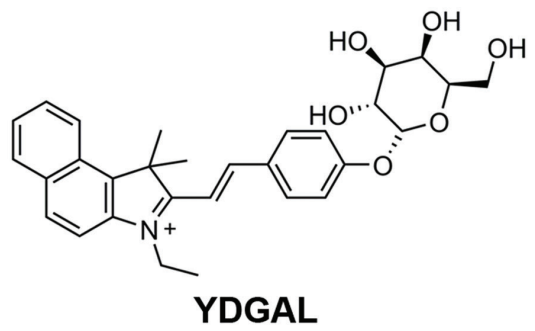

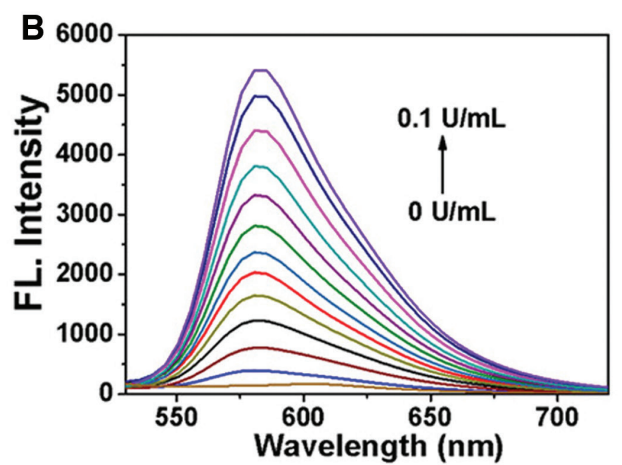

C

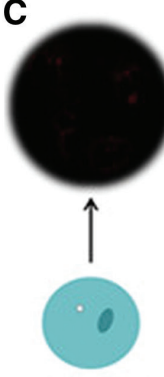

Control<smiles>C1CCC2CCC(C1)S2</smiles>

Control

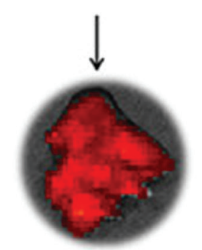

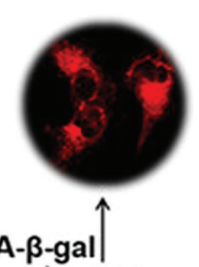
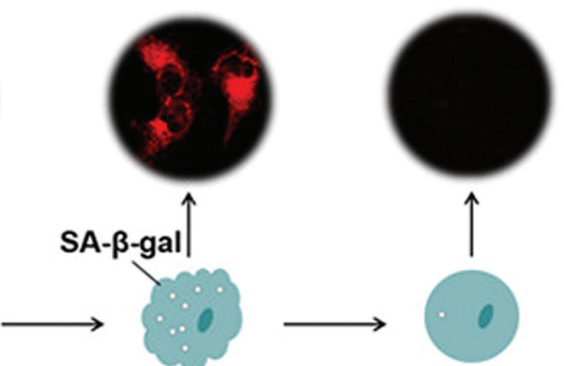

Senescent cell Treated cell

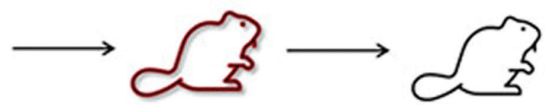

Aging mouse Treated mouse
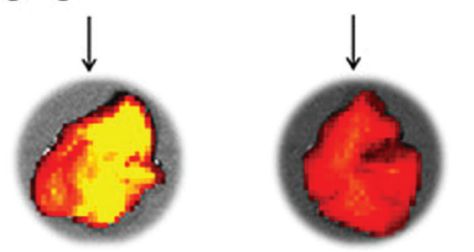

Figure 7 | (A) Chemical structure of YDGAL. (B) Fluorescence spectra of probe after the addition of $\beta$-gal. (C) Use of the probe to evaluate the therapeutic effects of anti-aging drugs. (B) and (C) Adapted with permission from Ref. [27]. Copyright 2021 American Chemical Society

The ability of HeckGal to detect $\beta$-gal in cells has been evaluated in normal human fibroblasts and senescent cancer cells including SK-Mel-103, 4T1, A549 and BJ cells. Importantly, the authors used two different models of senescence-related disease to investigate the ability of HeckGal to track the aging process in vivo, including an orthotopic breast cancer mouse model (BALB/cByJ mice) stimulated with palbociclib and a renal fibrosis mouse 


\section{fibrotic} kidney

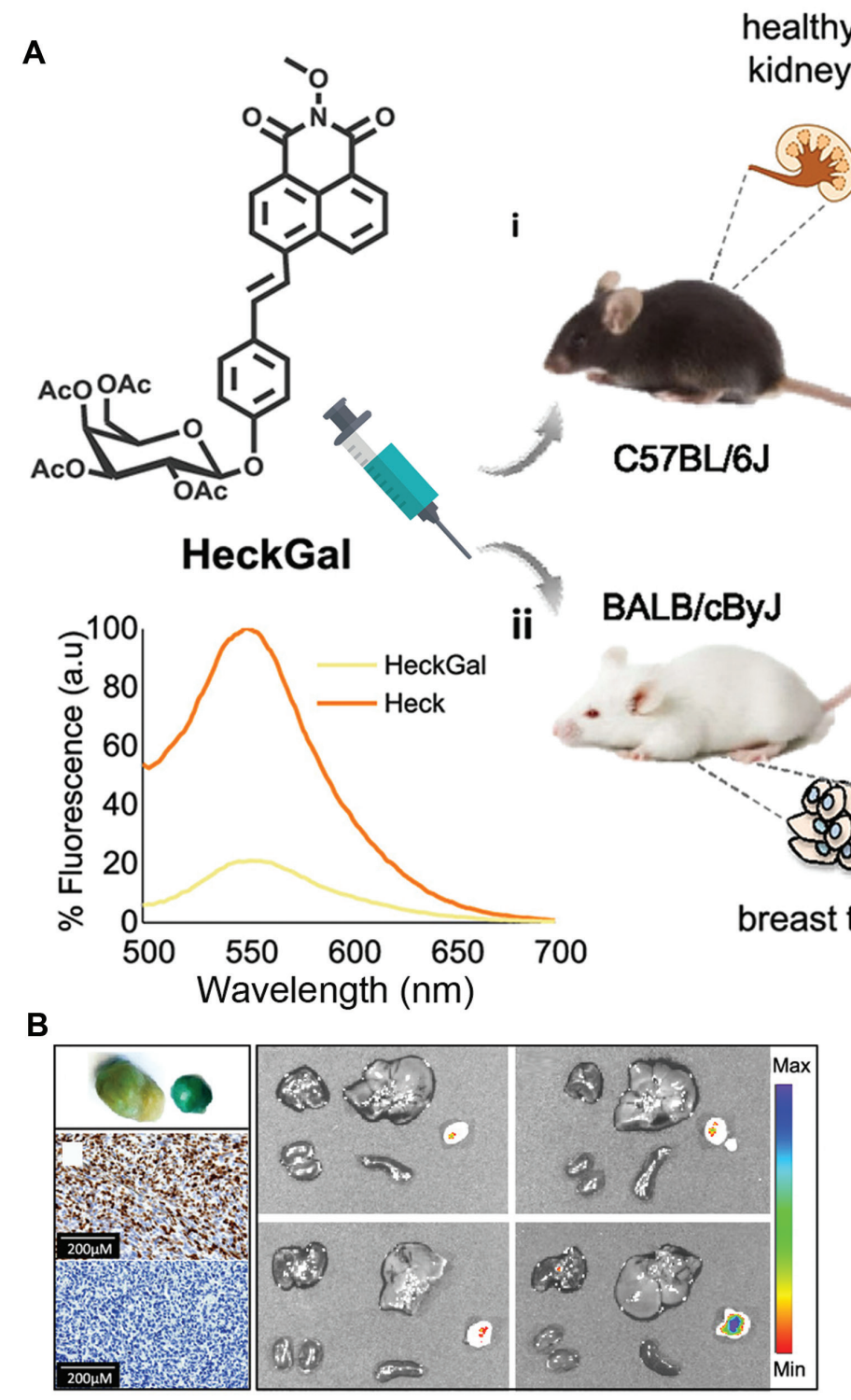

C

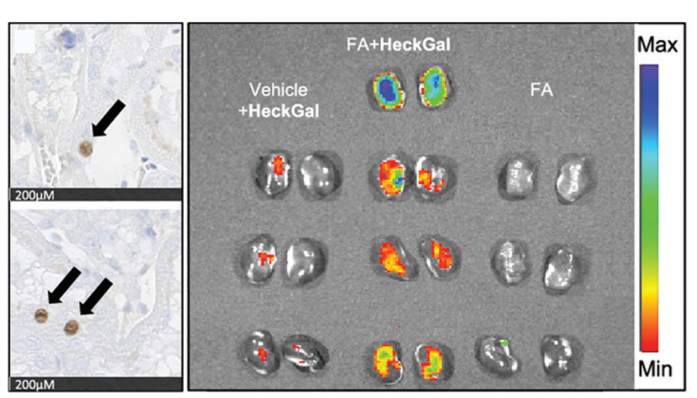

Figure 8 (A) Molecular design of the probe and the application of the probe in aged mice. (i) Kidney fibrosis in C57BL/6J male mice was induced by treatment with folic acid. (ii) BALB/CByJ female mice bearing 4 T1 breast cancer tumors were stimulated with palbociclib. The emission spectra of HeckGal and Heck are shown. (B) Images of tumors stained with the SA- $\beta$-Gal assay; immunohistochemical detection and fluorescence images of organs and tumors in BALB/CByJ female mice (ii) are shown. (C) Images of p21 immunostaining in kidney slides and fluorescence images of kidneys from C57BL/6J mice in the indicated groups (i). Scale bar: $200 \mu \mathrm{m}$. Adapted with permission from Ref. [28]. Copyright 2021 American Chemical Society.

model (C57BL/6J mice) induced by folic acid. As shown in Figure $8 B$, $C$, only senescent tumors emitted significant fluorescence in BALB/CByJ mice, and substantial fluorescence emission was observed in only the fibrotic senescent kidneys of FA-treated mice. Therefore, HeckGal enables unambiguous detection of senescence in vitro, as well as in tissues and tumors in vivo.

E. coli $\beta$-gal, a lacZ-encoded enzyme from bacteria, is frequently used as the SA- $\beta$-gal model protein in vitro $[29,30]$. However, unlike human $\beta$-gal, bacterial $\beta$-gal is not associated with senescence. To distinguish human
SA- $\beta$-gal from the $\beta$-gal from other species (such as $E$. coli $\beta$-gal), Li et al. have proposed a molecular-design strategy to build fluorescent probes by using structure-based steric hindrance adjustment to cater to various enzyme pockets [31]. The authors designed and synthesized a series of probes (KSL01-KSL12) differing in their steric hindrance around the $\beta$-D-galactosyl group (recognition group), thus providing a wide range of green-to-NIR color options for monitoring $\beta$-gal. Among those probes, KSL08-KSL12 exhibits desirable responses to only $A$. oryzae $\beta$-gal, thus enabling the first species-specific 
A<smiles>N#CC(C#N)=C1C=C(/C=C/c2ccc(OC3OC(CO)C(O)C(O)[C@H]3O)c(-c3nc4ccccc4s3)c2)CCC1</smiles>
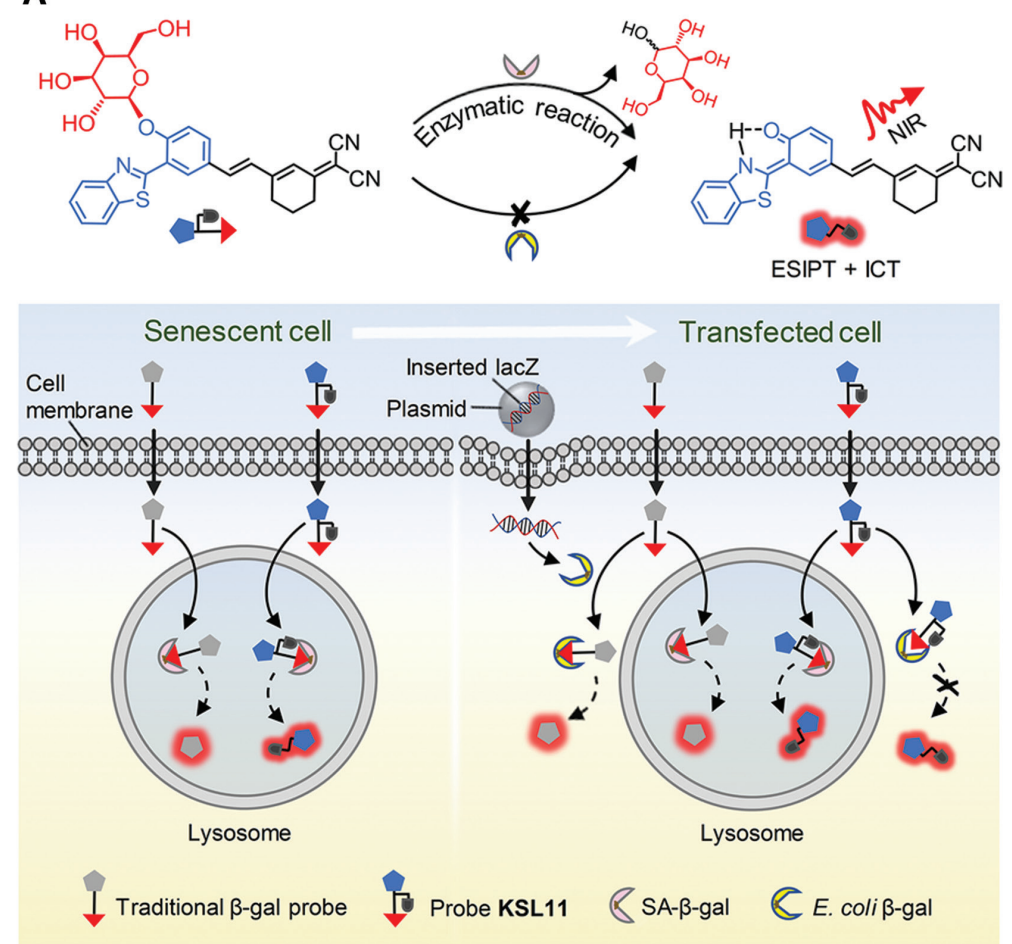
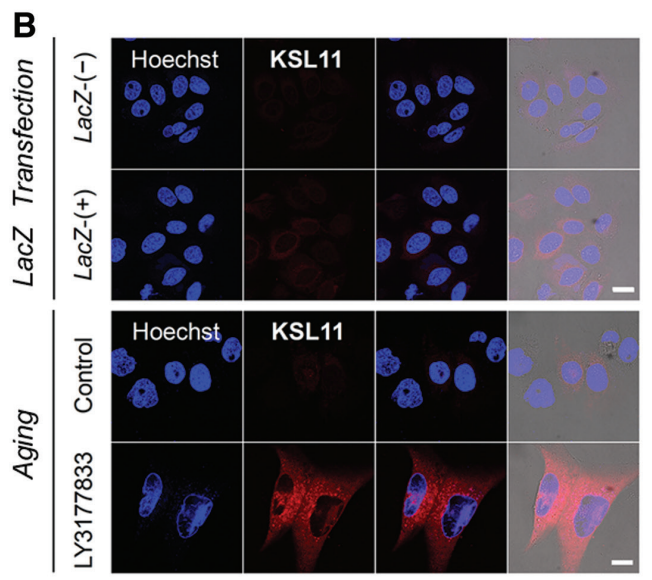

C

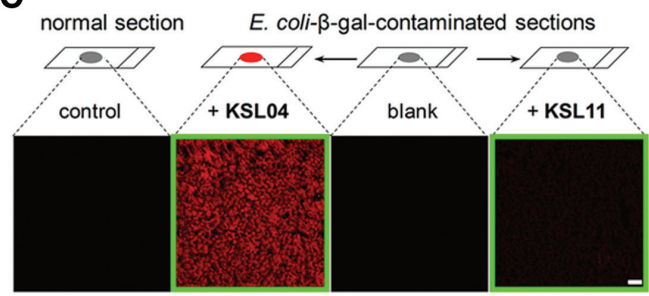

Figure 9 | (A) Chemical structure of the species-selective chemical probe KSL11 and schematic diagram of the application of this probe to distinguish human SA- $\beta$-gal from $E$. coli $\beta$-gal. (B) Representative images of HepG2 cells and Hep3B cells co-stained with $10 \mu M$ KSL11 and Hoechst. Scale bar: 20 Mm. (C) Confocal images of kidney sections contaminated by E. coli $\beta$-gal, incubated with KSL04 and KSL11. Adapted with permission from Ref. [31]. Copyright 2021 The Author(s).

identification of $\beta$-gal. KSL11 was selected as the representative probe among the first-generation species-selective probes (Figure 9A) and further investigated in senescent cells (MRC-5 cells), lacZ-transfected cells (293T and HepG2 cells) and tissue sections contaminated by $E$. coli $\beta$-gal (Figure 9B,C). KSL11 has been used to selectively monitor SA- $\beta$-gal without interference from bacterial $\beta$-gal in these samples. Importantly, with this probe, the authors have discovered that the content of SA- $\beta$-gal is gradually enhanced with age, and the kidney undergoes the most severe aging among organs during the aging process.

$\mathrm{Li}$ et al. have reported the NIR fluorescent probe KSL0401 for monitoring SA- $\beta$-gal activity [32]. This probe was constructed by grafting the coumarin-dicyanoisophorone hybrid fluorophore KSL0401-OH with a $\beta$-D-galactosyl group ( $\beta$-gal-activable trigger) (Figure 10A). KSL0401 releases the fluorophore KSL0401-OH, which exhibits a long NIR fluorescence wavelength $(706 \mathrm{~nm})$ and a large Stokes shift $(218 \mathrm{~nm})$ after being hydrolyzed by SA- $\beta$-gal (Figure 10B). Notably, KSL0401 displays a highly rapid response to $\beta$-gal (<80 s) (Figure 10B), which is better than that of most previously reported SA- $\beta$-gal probes. Additionally, KSL0401 shows good biocompatibility and has been used to monitor SA- $\beta$-gal activity in various senescent cell types, including $\mathrm{H}_{2} \mathrm{O}_{2}$-induced senescent MRC-5 cells, MLN4924-induced senescent HepG2 cells and A549 cells (Figure 10C), thus indicating the feasibility of using KSL0401 to evaluate the degree of senescence.

Lin et al. have developed the fluorescent probe TR-G for the detection of $\beta$-gal [33]. The authors first synthesized the new fluorophore Rho by replacing the phenethylamine moiety of rhodamine B with naphthol, then introduced a $\beta$-D-galactose group into the fluorophore to build the probe TR-G (Figure 11). After the addition of $\beta$-gal, TR-G exhibited a marked increase in fluorescence intensity at $638 \mathrm{~nm}$. TR-G also exhibits two-photon properties, thereby indicating that the probe is suitable for monitoring $\beta$-gal in deep tissues. The activity of $\beta$-gal in living cells, organs and tissues has been detected effectively with TR-G. More importantly, TR-G has been successfully used to monitor $\beta$-gal in tumor tissue with deep penetration (up to $120 \mu \mathrm{m}$ in tissues) through two-photon microscopy. TR-G has also been applied to monitoring the activity of $\beta$-gal in vivo in tumors with $\beta$-gal overexpression in a mouse model.

Yang et al. have developed the $\beta$-gal-responsive molecule Comp. 1 (TPE-ETh-R-GFFY(gal)- ERGD), which is based on the novel AIEgen TPEETh-R (Figure 12), emits strong red fluorescence and generates cytotoxic ROS under light excitation in the aggregated state [34]. 
A

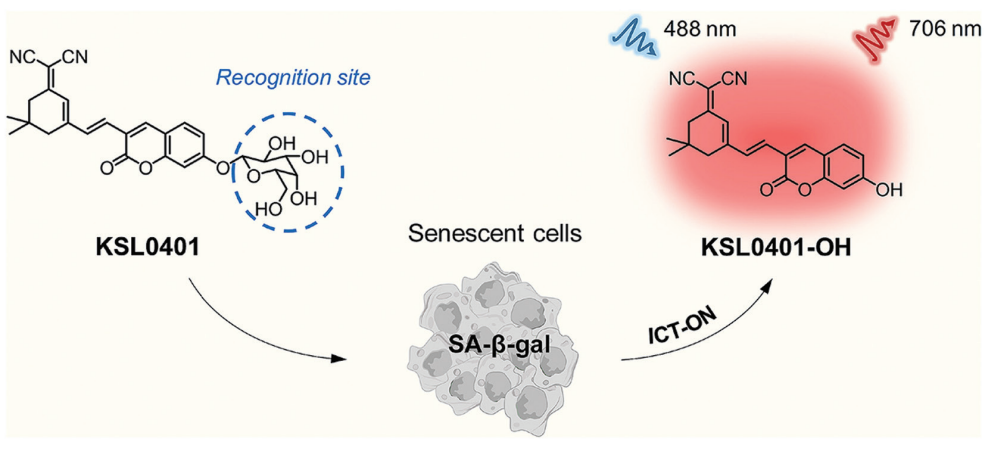

B
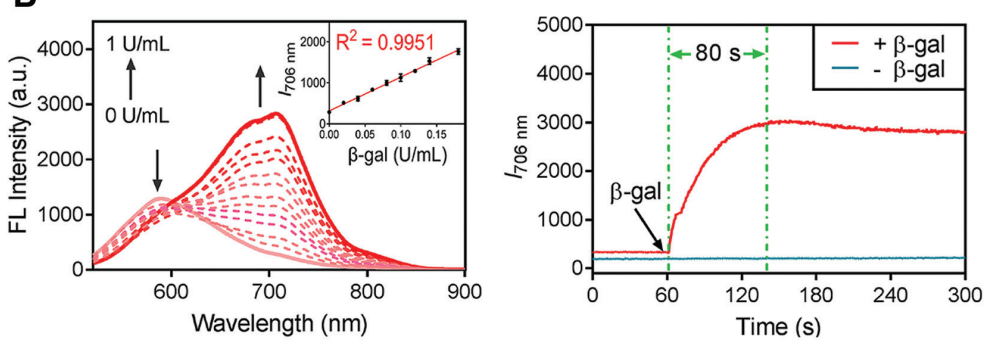

C
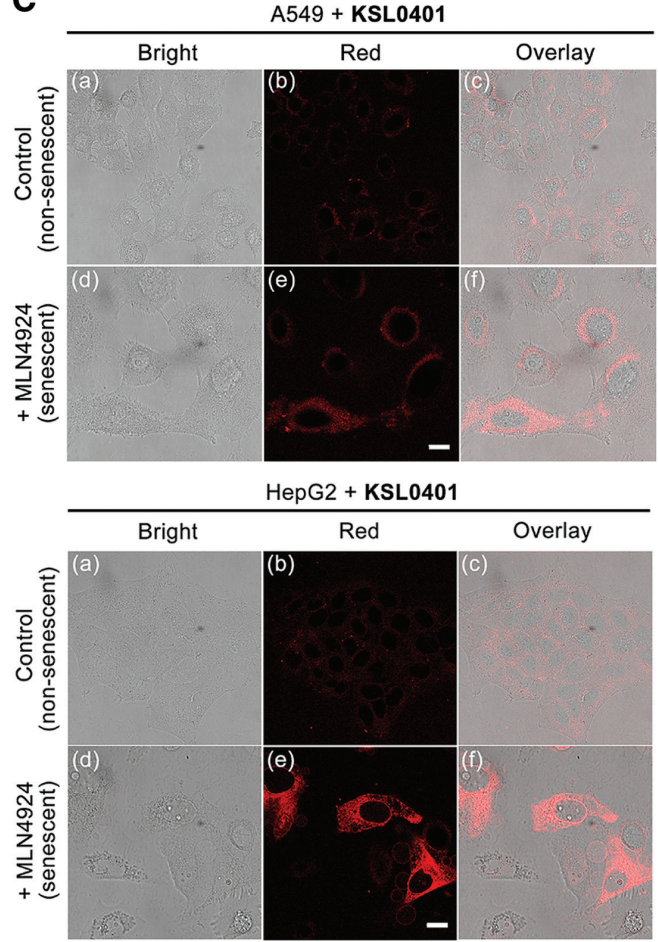

Figure 10 | (A) Molecular design of KSL0401 and its mechanism for SA- $\beta$-gal detection. (B) Emission spectra and kinetic analysis of KSL0401 after the addition of $\beta$-gal. (C) Representative confocal images of young cells and drug-induced senescent cells after incubation with KSL0401. Scale bar: 30 m. Reprinted with permission from Ref. [32]. Copyright 2021 Elsevier.

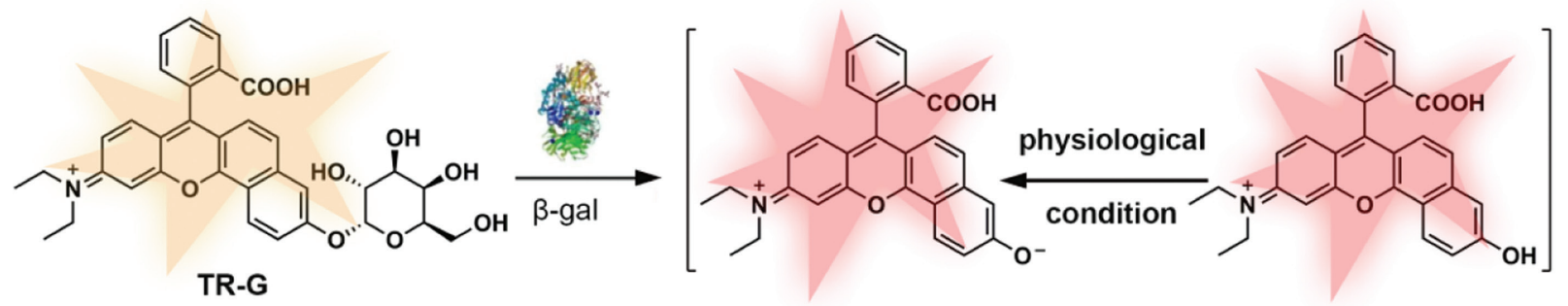

Figure 11 | Molecular design and the proposed detection mechanism for SA- $\beta$-gal of TR-G.

Comp. 1 can be transformed into TPE-ETh-R-GFFYERGD after hydrolysis by $\beta$-gal, thus leading to the production of a supramolecular assembly with remarkable fluorescence and ROS generation. Comp. 1 can specifically identify cisplatin-induced senescent Hela cells with strong red fluorescence. Additionally, Comp. 1 under light irradiation selectively produces ROS, thereby removing senescent HeLa cells under light irradiation. Consequently, Comp. 1 has the potential to be used to monitor and remove senescent cancer cells.

Bhuniya et al. have developed the $\beta$-gal fluorescent probe, SRP, to track senescence (Figure 13) [35]. SRP displays faint fluorescence in spirocyclic form. After the addition of $\beta$-gal, a marked increase $(\sim 27$-fold) in fluorescence intensity at $545 \mathrm{~nm}$ relative to the probe has been observed. SRP exhibits high reactivity to $\beta$-gal within a $\mathrm{pH}$ range of 5-9 and consequently has the potential to evaluate $\beta$-gal activity in cell lysosomes. In addition, the probe can target lysosomes. More importantly, SRP has high biocompatibility and can distinguish senescent from young human umbilical vein cells, thus confirming the feasibility of using SRP to assess cellular senescence.

ROS, such as the hydrogen peroxide $\left(\mathrm{H}_{2} \mathrm{O}_{2}\right)$, the hydroxyl radical $(\mathrm{OH} \bullet)$, the superoxide anion $\left(\mathrm{O}_{2}{ }^{-}\right)$and hypochlorous acid $(\mathrm{HOCl})$, act as intracellular signals 


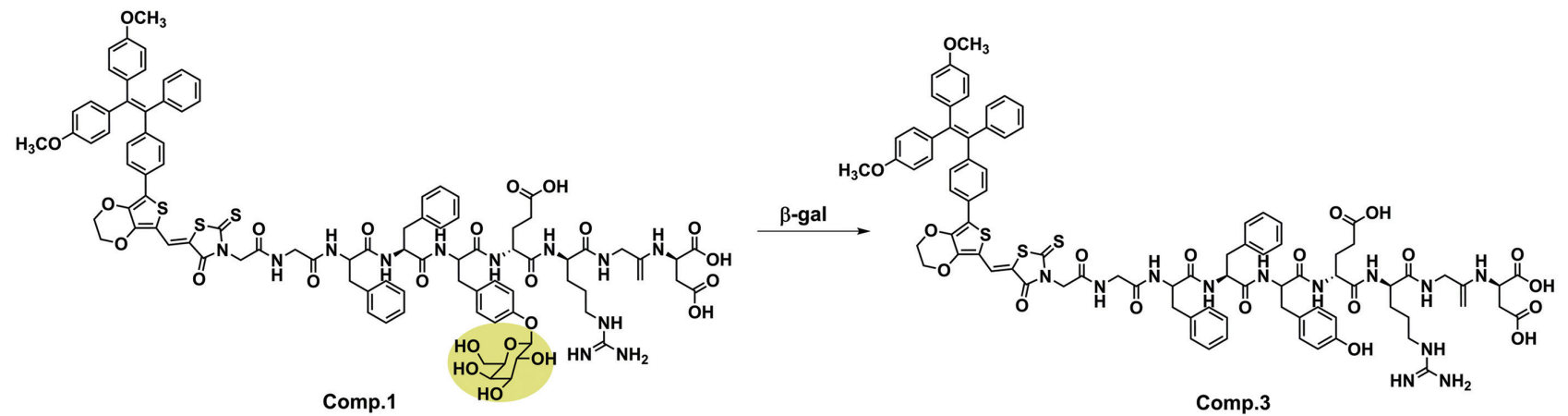

Figure 12 | Chemical structure of the probe Comp. 1 and the proposed sensing mechanism.

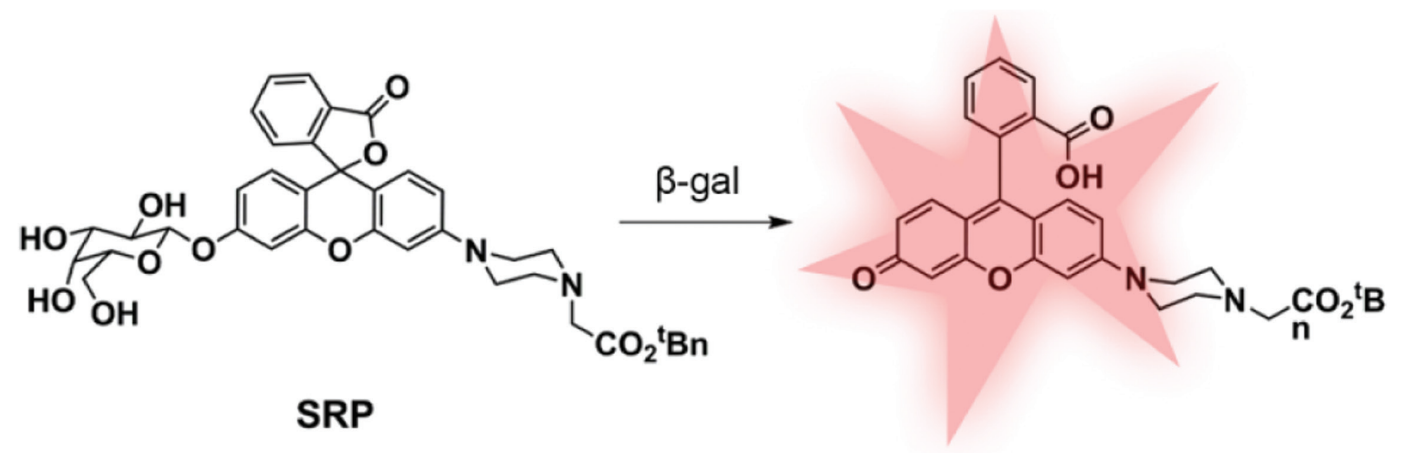

Figure 13 | Molecular design of SRP and its proposed detection mechanism.

and growth stimulants. However, excess ROS cause oxidative damage to DNA and further promote stress-induced premature senescence in cells [36]. Typically, the ROS levels increase in various types of cancers and cellular senescence [37]. Mizuta et al. have developed two fluorescent probes to detect oxidative stress and the activity of $\beta$-gal to evaluate stress-induced premature senescence [38]. The authors first synthesized six candidate fluorescent probes 1a-f for ROS detection and screened the optimal probe 1a (Figure 14A,B). Probe 1a displayed a ratiometric response to $\mathrm{ROS}\left(\mathrm{H}_{2} \mathrm{O}_{2}, \mathrm{OH} \bullet\right.$ and $\mathrm{O}_{2}^{--}$) and was further used to image the accumulation of ROS in living cells. In addition, the authors designed and synthesized the fluorescent probe CBT- $\beta$ Gal 4 to monitor $\beta$-gal activity. CBT- $\beta$-Gal 4 was used to monitor $\beta$-gal activity during oxidative-stress-induced senescence, and Hela cells stimulated by $\mathrm{H}_{2} \mathrm{O}_{2}$ exhibited marked fluorescence. Thus, probes $1 \mathrm{a}$ and CBT- $\beta$ Gal 4 can be used to monitor the increased levels of ROS and $\beta$-gal activity in drug-induced senescent cells (Figure 14C).

$\mathrm{Li}$ et al. have developed the NIR fluorescent probe QX-B for the detection of $\mathrm{H}_{2} \mathrm{O}_{2}$ [39]. The probe was constructed on the basis of a quinolinium-xanthene fluorophore with long absorption $(725 \mathrm{~nm})$ and emission $(772 \mathrm{~nm})$ wavelengths. Borated ester-caged
QX-B is non-fluorescent, because the intramolecular charge-transfer process was blocked (Figure 15A). After reaction with $\mathrm{H}_{2} \mathrm{O}_{2}$, the borate ester unit of the probe was removed, and the intramolecular charge-transfer process was restored, thus leading to a pronounced fluorescence signal. The probe was used to monitor exogenous and endogenous $\mathrm{H}_{2} \mathrm{O}_{2}$ in various cells, including HCT116, 4T1 and HeLa cells. Furthermore, the probe successfully enabled visual tracking of $\mathrm{H}_{2} \mathrm{O}_{2}$ in zebrafish and in mice (Figure 15B,C).

$\mathrm{Yu}$ et al. have developed a mitochondria-targetable NIR fluorescent probe (Mito-Bor) for tracking of $\mathrm{H}_{2} \mathrm{O}_{2}$ in vitro and in vivo [40]. The probe consists of three moieties comprising an NIR fluorophore (azoBODIPY), a $\mathrm{H}_{2} \mathrm{O}_{2}$-responsive unit (aryl borate) and a mitochondrial targeting group (triphenylphosphonium cation) (Figure 16A). Mio-Bor is highly sensitive to $\mathrm{H}_{2} \mathrm{O}_{2}$, and the limit of detection has been calculated to be $23 \mathrm{nM}$. Moreover, the probe specifically targets mitochondria and can be used to monitor $\mathrm{H}_{2} \mathrm{O}_{2}$ in live cells (Figure 16B,C). Using Mio-Bor, the authors have successfully monitored the changes in $\mathrm{H}_{2} \mathrm{O}_{2}$ levels in mouse models of pulmonary fibrosis induced by bleomycin (Figure 16D).

Many studies have documented that cellular senescence can cause significant changes in the intracellular 
A<smiles>Oc1ccccc1-c1nc2ccccc2s1</smiles>

$1 \mathrm{a}$<smiles>OCC1OC(Oc2cc(Cl)ccc2-c2nc3ccccc3s2)C(O)C(O)C1O</smiles>

CBT- $\beta$-Gal 4

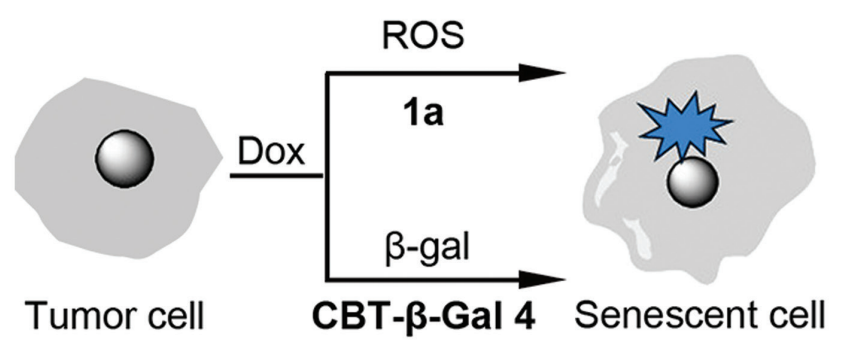

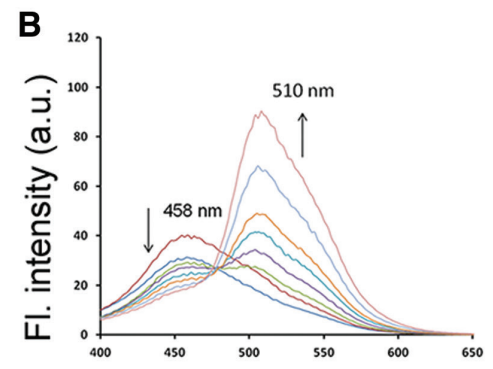

Wavelength $(\mathrm{nm})$

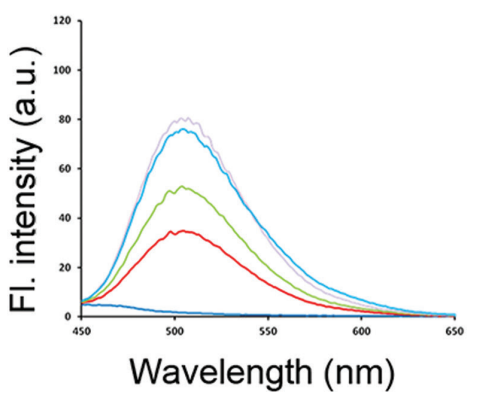

C
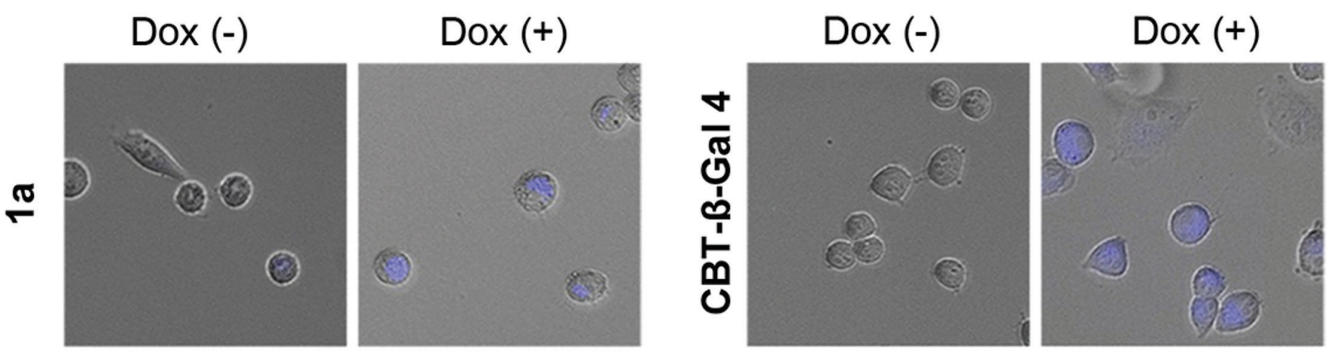

Figure $14 \mid$ (A) Chemical structures of $1 \mathbf{a}$ and CBT- $\beta$-Gal 4. (B) Emission spectra of $\mathbf{1 a}$ after the addition of $\mathrm{H}_{2} \mathrm{O}_{2}$ and of CBT- $\beta$-Gal 4 after reaction with $\beta$-gal. (C) Images of $\mathbf{1 a}$ and CBT- $\beta$-Gal $\mathbf{4}$ in HeLa cells incubated with or without doxorubicin (Dox). Adapted with permission from Ref. [38]. Copyright 2021 American Chemical Society.

microenvironment [41, 42]. Polarity, a microenvironmental factor, is considered to play an important role in multiple cellular processes including proliferation, cell differentiation and regulation of the immune system [43]. Li et al. have developed six curcumin-based fluorescent probes (KSLP1-6) for the detection of polarity [44]. Thru analysis of the detection ability of all probes, KSLP1 was identified for its high selectivity to polarity and high biocompatibility. The probe KSLP1 exhibited a significant enhancement (42-fold) in fluorescence intensity at $\sim 700 \mathrm{~nm}$, as the polarity changed from $\Delta f=0.397$ to $\Delta f=0.367$ (Figure 17A,B). The polarity in various cells, including 293T, HL-7702, A549, HepG2 and Hep3B cells, has been evaluated with KSLP1. Moreover, KSLP1 has been used to monitor lysosomal polarity in LY3177833-induced senescent Hep3B cells and replication-induced senescent MRC-5 cells. The polarity in lysosomes increases with senescence (Figure 17C-E). Furthermore, the polarity of Caenorhabditis elegans ( $C$. elegans) at various ages (from 2 days old to 10 days old) has been visualized with KSLP1, and the overall body polarity has been found to increase with age. The authors have proposed a potential mechanism for the variation in polarity in lysosomes with senescence, in which the activity of pH-dependent hydrolases is decreased by the de-acidification of lysosomes, thereby promoting proteolysis of these lysosomal enzymes and resulting in further production of hydrophilic substrates (Figure 17F).

Lysosomes, ubiquitous and dynamic organelles, receive and degrade macromolecules from various pathways including secretory autophagic, endocytic and phagocytic membrane-trafficking pathways [45]. The $\mathrm{pH}$ value of lysosomes ranges from 4.5 to 5.5 in the normal cellular state, which is maintained by proton-pumping vacuolar ATPases. Studies have indicated altered v-ATPase activity and lysosomal $\mathrm{pH}$ dysregulation in cellular senescence $[45,46]$. Miao et al. have reported the small-molecule fluorescent probe SGJ, which can selectively and sensitively monitor $\mathrm{pH}$ with a 
A

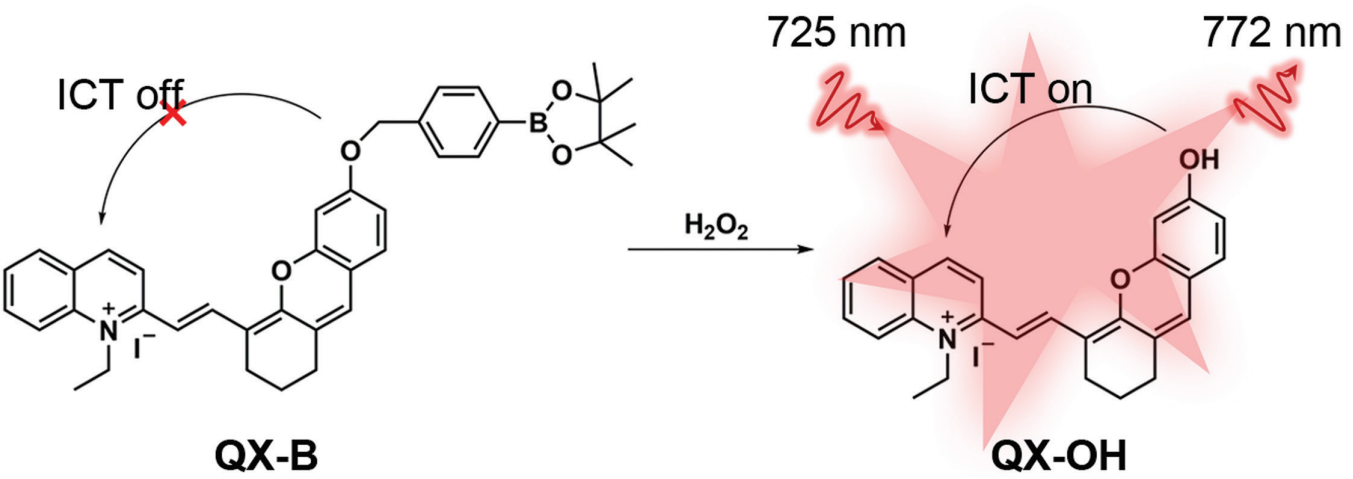

B
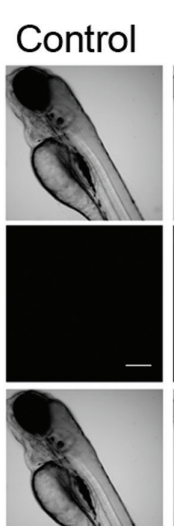
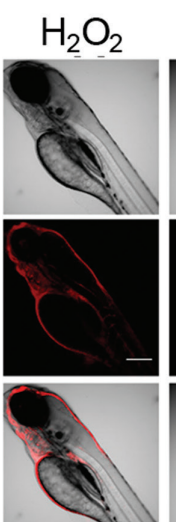
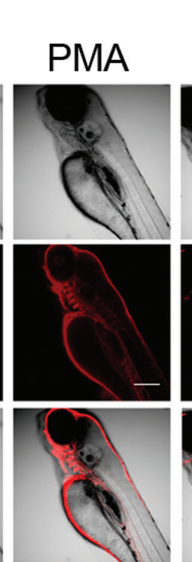

PMA

NAC

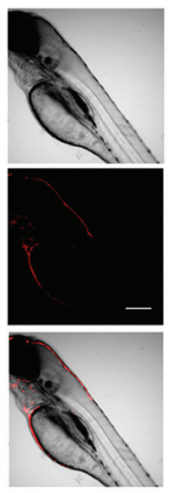

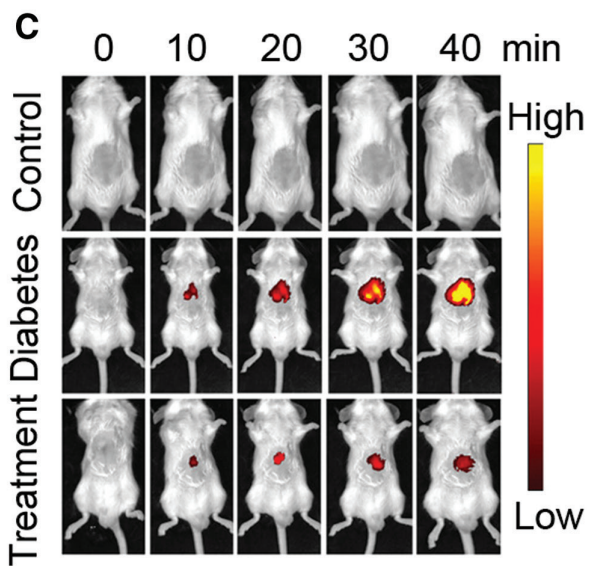

Figure 15 | (A) Chemical structure of QX-B and sensing mechanism. (B) Confocal images of zebrafish stained with QX-B. Scale bar: $200 \mu$ m. (C) Fluorescence imaging of mice from the indicated groups after the injection of QX-B. (B) and (C) Adapted with permission from Ref. [39]. Copyright 2021 American Chemical Society.

rapid response $[47,48]$. SGJ was designed and synthesized on the basis of a novel fluorophore comprising an imidazo[1,5-a]pyridine derivative, which has shown good biological activity in anti-inflammation and has been widely used to develop medicines (Figure 18A). The probe shows a rapid response, good reversibility, high selectivity and sensitivity to $\mathrm{pH}$ and is able to target lysosomes (Figure 18B). Interestingly, the authors have discovered that SGJ suppresses cellular senescence and improves the viability of senescent cells. After treatment with SGJ, the acidic vacuoles in senescent cells significantly increase, on the basis of staining with acridine orange, an indicator of changes in lysosomal $\mathrm{pH}$ (Figure 18C). Furthermore, SGJ inhibits the decrease in $\mathrm{H}^{+}$concentrations in lysosomes; increases the expression of the lysosome-associated membrane proteins LAMP1 and LAMP2 (Figure 18D); and restores lysosomal activity in senescent cells, thereby suppressing senescence.

Oh et al. have performed high-throughput screening based on the diversity-oriented fluorescence library approach [49]. Using this platform to screen 6417 compounds (fluorescent probes), the authors found that the fluorescent probe CyBC9 (Figure 19A,B) specifically stains senescent human mesenchymal stromal cells (MSCs). CyBC9 exhibits high cellular uptake, and high sensitivity and specificity in monitoring senescent MSCs in both early and late stages of senescence. Moreover, CyBC9 stains live senescent MSCs in microcarrier-based culture. To study the mechanism of CyBC9's detection of senescent cells, the authors performed colocalization assays using CyBC9, which revealed that the probe accumulates in the mitochondria, according to the high Pearson correlation coefficient values. Furthermore, using carbonyl cyanide $m$-chlorophenyl hydrazone (CCCP) to decrease the membrane potential of mitochondria by inducing uncoupling of mitochondria in living cells, the authors found that CCCP-treated cells and senescent cells are specifically stained by СyBC9, thus suggesting that CyBC9 selectively stains senescent cells because of the decrease in membrane potential of mitochondria with senescence. 
A
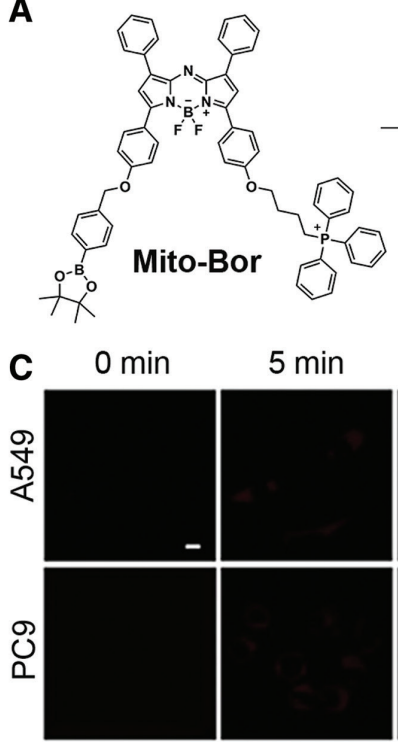

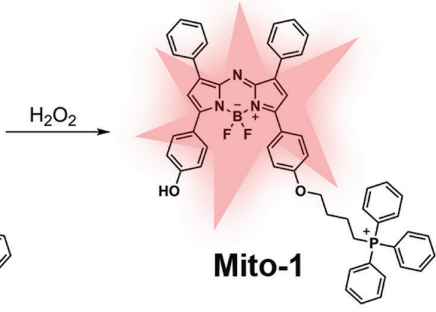

B

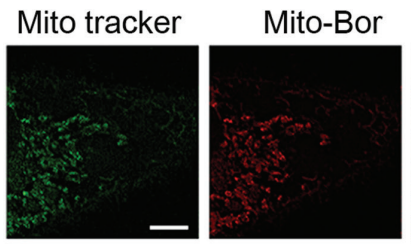

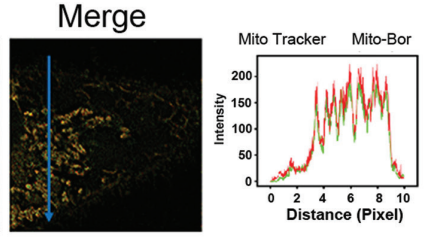

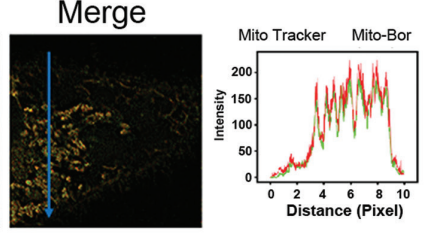

D

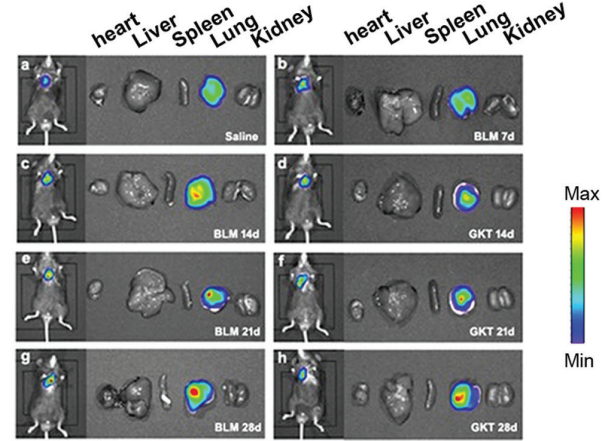

Figure 16 ( (A) Chemical structure of Mito-Bor and the sensing mechanism. (B) Co-localization experiments with A549 cells stained by Mito tracker and Mito-Bor. Scale bar: $2 \mu \mathrm{m}$. (C) Confocal images of A549 cells and PC 9 cells after incubation with Mito-Bor. Scale bar: $10 \mu \mathrm{m}$. (D) In vivo fluorescence imaging of mice (model of pulmonary fibrosis) treated with the probe via an intratracheal spray. (B-D) Adapted with permission from Ref. [40]. Copyright 2021 American Chemical Society.

\subsection{Fluorescent probes for precise diagnosis of aging, in the time and space dimensions}

Most evaluations of senescence and diagnosis of aging have focused on monitoring the levels of single agerelated biomarkers in the resting state. However, the non-specificity of current senescence-associated biomarkers and the existence of different senescence programs have strongly limited these methods.

Previously described fluorescent probes for monitoring SA- $\beta$-gal to track cellular senescence have reported on only a single dimension: the accumulation of SA- $\beta$ gal in lysosomes. However, endogenous $\beta-$ gal is closely associated not only with senescence but also with several other biological processes, and thus single-marker levels are insufficient to fully evaluate aging. Recently, Guo et al. have developed two-dimensional fluorescent probes (KSA01 and KSA02) to precisely track senescence [50]. De-acidification has been reported as a unique feature of lysosomes in senescent cells. Thus, the authors have proposed a two-dimensional detection strategy that relies on the monitoring of SA- $\beta$-gal activity in combination with the detection of $\mathrm{pH}$ in the lysosomal microenvironment. Both probes were designed on the basis of merocyanine-based fluorescent dyes modified by carboxyl groups to achieve sensitivity within the $\mathrm{pH}$ range of lysosomes. These $\mathrm{pH}$-sensitive dyes contain a $\beta$-D-galactosyl group as the $\beta$-gal-responsive site (Figure 20A). The ability to simultaneously monitor $\beta$-gal activity and the local microenvironment of the probes was first verified in vitro (Figure 20B). Using the probes, the authors have successfully distinguished the SA- $\beta$-gal in senescent HL-7702 cells from cancer-related endogenous $\beta$-gal in SKOV-3 cells. As shown in Figure 20C, KSA02-stained senescent cells mainly display red fluorescence, whereas KSA02-stained SKOV-3 cells exhibit green fluorescence, thus indicating the ability of KSA02 to distinguish SA- $\beta$-gal from cancer-associated $\beta$-gal. Furthermore, the visual detection of SA- $\beta$-gal by KSA01 and KSA02 in kidney tissues with different degrees of aging has been achieved (Figure 20D).

Examination of the stress response capacity (SRC) holds promise in the dynamic assessment of aging. Thiophenol (PhSH), a highly toxic chemical substance, can induce cellular oxidative stress and lead to increased levels of ROS, such as HOCl. Accordingly, $\mathrm{PhSH}$ and $\mathrm{HOCl}$ act as the stressor and the stress product, and the sequential and logical detection of $\mathrm{PhSH}$ and $\mathrm{HOCl}$ is crucial to detect the evolution of stress and evaluate the SRC. Very recently, Guo et al. developed the first SRC fluorescent probe, ROKS (Figure 21A), a dual-channel fluorescent probe enabling sequential, logical detection of $\mathrm{PhSH}$ and $\mathrm{HOCl}$ [51]. ROKS displays faint fluorescence, whereas a significant emission peak at $534 \mathrm{~nm}$ is observed upon reaction with $\mathrm{PhSH}$. After continued addition of $\mathrm{HOCl}$, this emission peak $(534 \mathrm{~nm})$ disappears, and simultaneously a red emission peak at $602 \mathrm{~nm}$ forms (Figure 21B,C). Thus, the fluorescence ratio of both channels $\left(I_{\text {red }} / I_{\text {green }}\right)$ can be used to precisely evaluate the SRC in individuals. With the dual-channel fluorescent probe ROKS, the changes 
A<smiles>CCN(CC)c1ccc(/C=C/C2=CC(/C=C/c3ccc(O)cc3)=[P-](F)(F)O2)cc1</smiles>

B

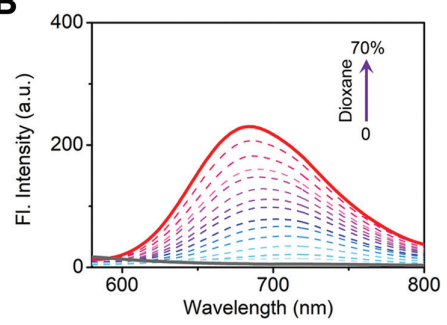

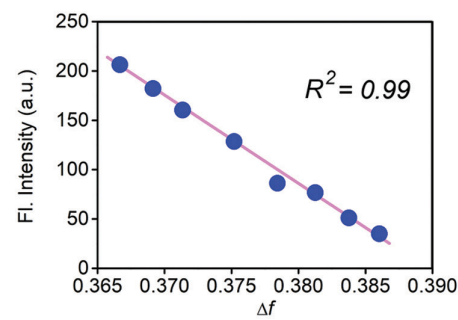

C

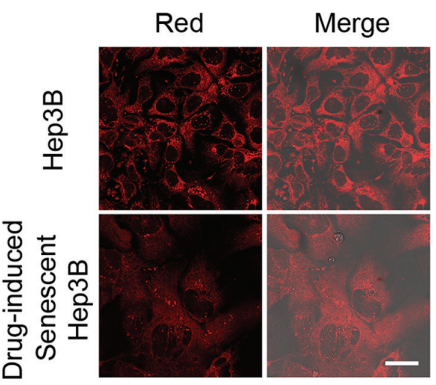

D

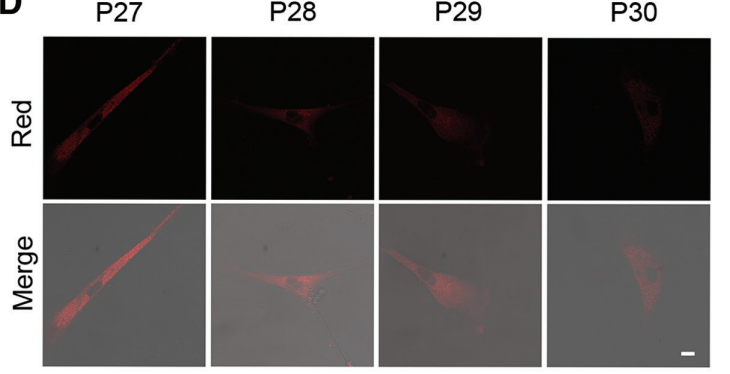

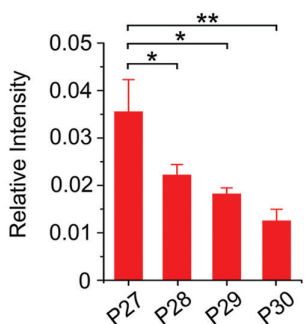

E

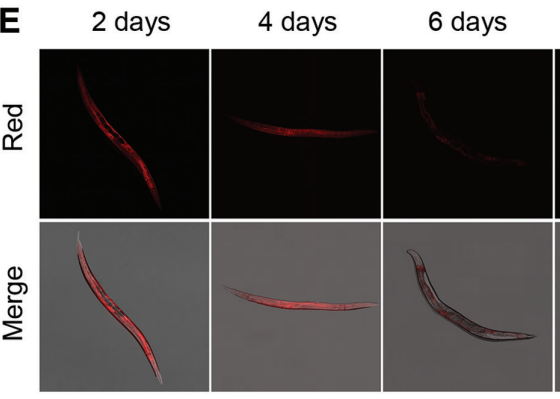
8 days 10 days

$F$
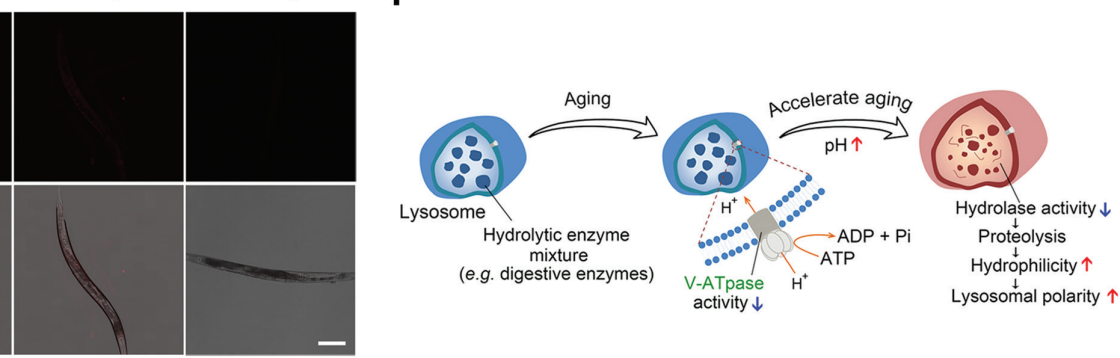

Figure $17 \mid$ (A) Chemical structure of KSLP1. (B) Emission spectra of KSLP1 in mixtures of dioxane and water (dioxane from 0\% to 70\%) and the relationship between the fluorescence intensity at $\sim 700 \mathrm{~nm}$ and the orientation polarizability $(\Delta f)$. (C) Confocal images of Hep3B cells and senescent Hep3B cells incubated with KSLP1. Scale bar: $25 \mu \mathrm{m}$. (D) Confocal images of MRC-5 cells incubated with KSLP1. Scale bar: $25 \mu \mathrm{m}$. (E) Fluorescence images of $C$. elegans at different ages (from 2 days old to 10 days old), stained with KSLP1. Scale bar: $200 \mu \mathrm{m}$. (F) Schematic illustration of proposed variations in lysosomal polarity in aging. (B-F) Reprinted with permission from Ref. [44]. Copyright 2021 Elsevier.

in SRC, as a function of aging within C. elegans at different ages, has been monitored. As shown in Figure 21D, the SRC of young $C$. elegans is higher than that of aged C. elegans after stimulation with $\mathrm{PhSH}$, thereby suggesting that SRC decreases with aging. These results have confirmed that the SRC-based strategy is particularly useful to precisely track the aging process from a dynamic perspective.

\section{CONCLUSIONS AND PERSPECTIVES}

As described above, fluorescent probes for the diagnosis of aging are important in aging research and healthspan evaluation. We have summarized the advances in various types of small-molecule fluorescence-based probes used for the diagnosis of aging. These optical probes can be divided into two categories according to various age-related biomarkers, including enzymes ( $\beta$-gal, $\alpha$-Fuc, GPx and SIA) and other markers (small active molecules and microenvironmental factors), and the novel detection strategies have expanded from the time dimension to the space dimension. Among these probes, TP-SS, YDGAL, HeckGal and TR-G are two-photon probes for aging diagnosis, which can be used for in vivo biological imaging. KSL11, the first-generation species-selective fluorescent probe for SA- $\beta$-gal, has enabled the first species-selective identification of human SA- $\beta$-gal. In addition, Sia-RQ and NIR-BG2 can label proteins after activation, thereby allowing for in situ imaging of age-related biomarkers. Moreover, compared with probes that monitor the single-dimensional markers to evaluate the aging process, KSA01-02 and ROKS, designed to detect the dimensions of space and time, can be used to track aging more precisely. Generally speaking, researchers have developed diverse fluorescent probes based on different biomarkers and strategies to diagnose aging, and have made exciting progress. 
A<smiles>CCCCc1nc(Cl)c2cc(C(=O)O)ccn12</smiles>

SGJ
B

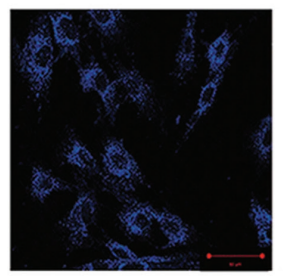

Lysotracker

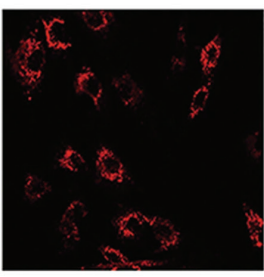

Merge

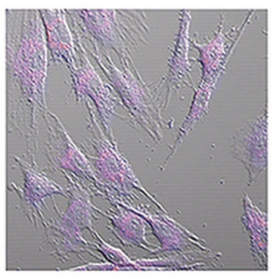

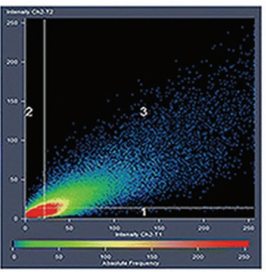

D

C

\section{PDL 5 $12 \mathrm{~h}$}
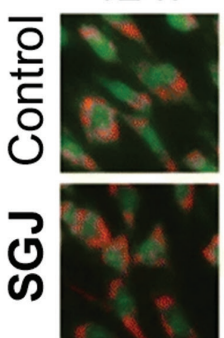

PDL 20
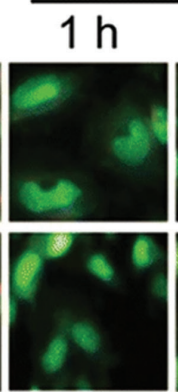
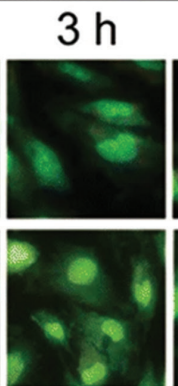

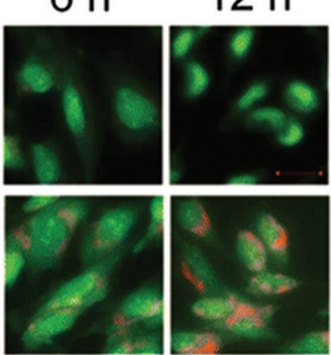

SGJ $\frac{6 \mathrm{~h}}{-+} \frac{12 \mathrm{~h}}{-+} \frac{24 \mathrm{~h}}{-+} \frac{48 \mathrm{~h}}{-+}$

LAMP1 $-\infty \infty \infty \infty \infty$

LAMP2 $\because-2=$

ACTB

Figure 18 | (A) Chemical structure of the probe SGJ. (B) SGJ co-located with lysosome trackers. (C) Fluorescence images of young BMSCS (population doubling level, PDL 5) and senescent BMSCS (PDL 20) stained with acridine orange (AO). (D) Expression levels of LAMP1 and LAMP2, analyzed by western blotting with $\beta$-actin as a loading control. BMSCs were treated with $\mathbf{S G J}(20 \mu \mathrm{M})$ for different times.

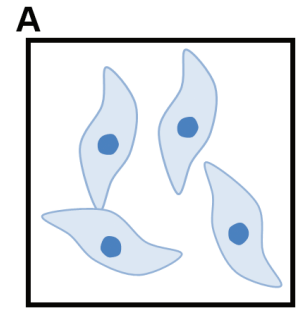

Proliferative heMSC

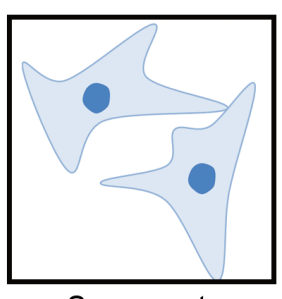

Senescent heMSC

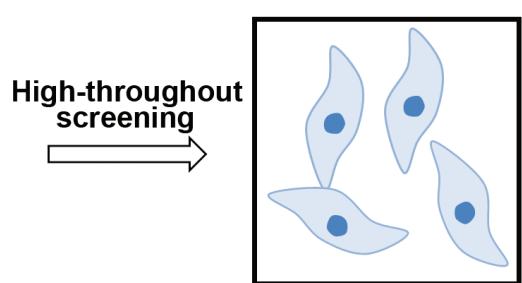

Proliferative heMSC

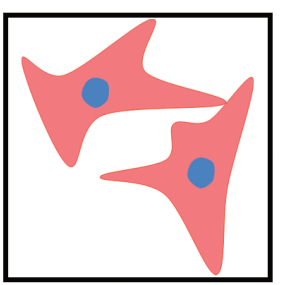

Senescent heMSC<smiles>[B]C1=[N+](CCCNC(=O)OC(C)(C)C)c2ccccc2C1(C)C=CC=CC=C1N(CCCNC(=O)Cc2c(Cl)cccc2Cl)c2ccccc2C1(C)C</smiles>

Figure $19 \mid$ (A) Senescence-specific probes for MSCs screened through a diversity-oriented fluorescence library approach and (B) chemical structure of СуВС. 
A
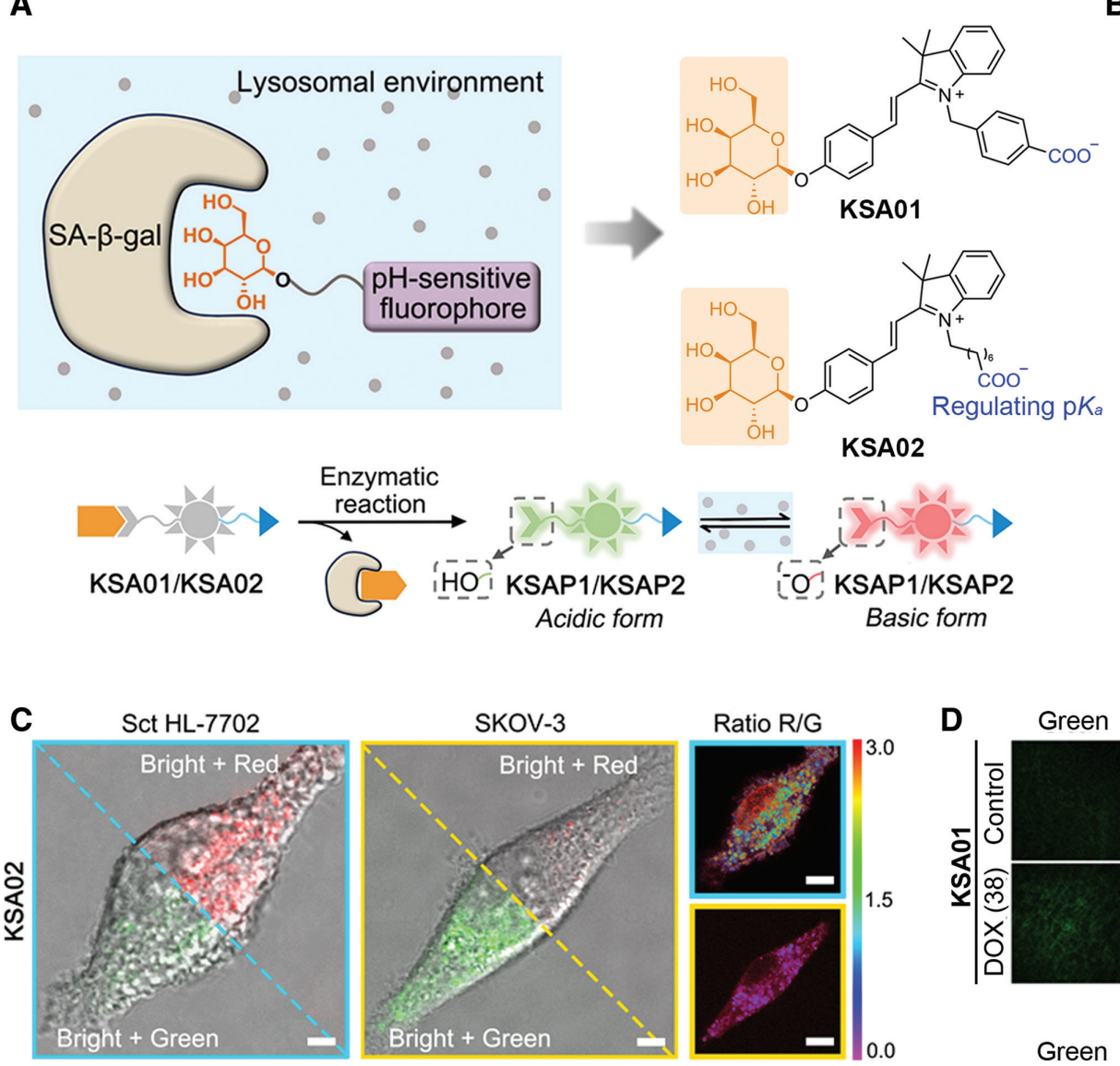

B
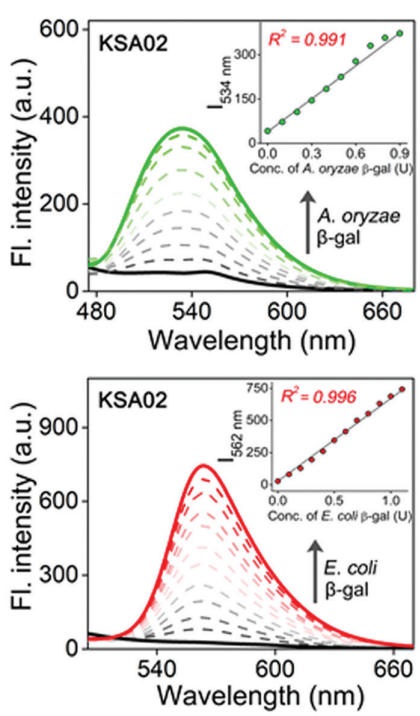
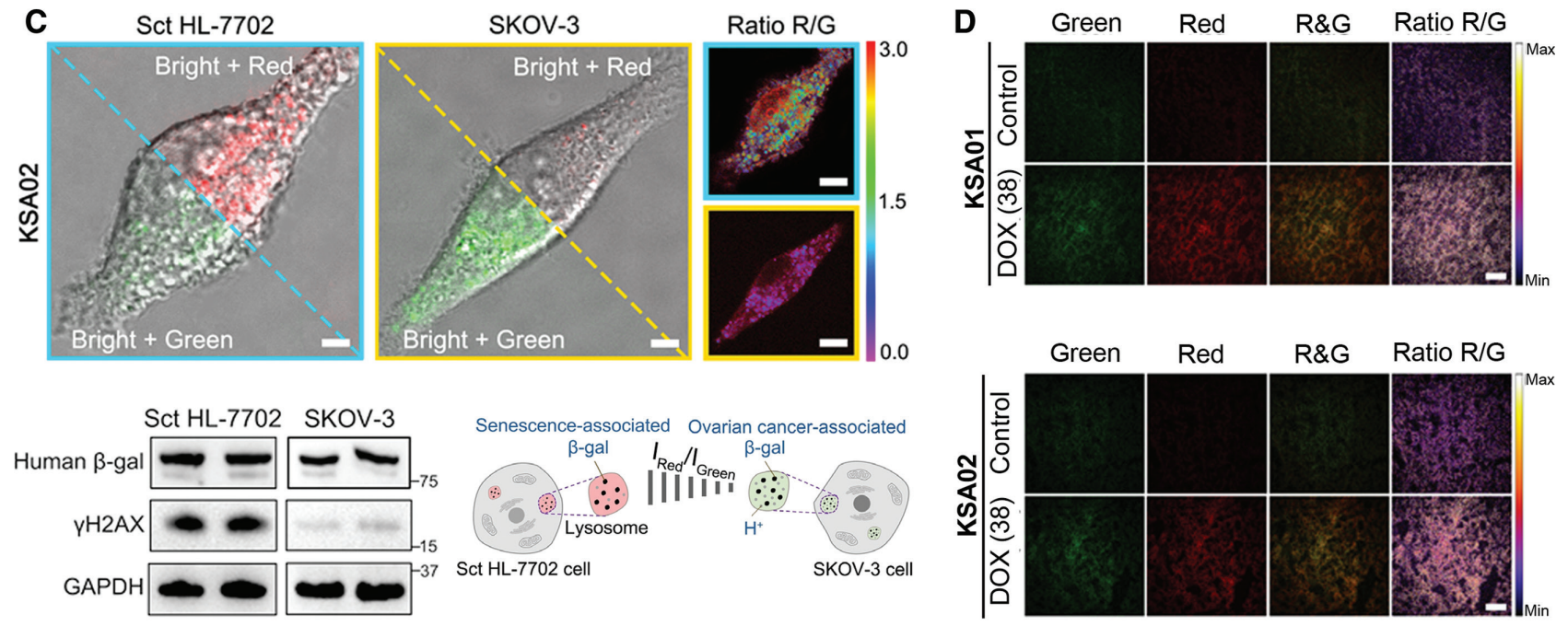

Figure $20 \mid$ (A) Schematic diagram of the molecular design of two-dimensional fluorescent probes for tracking aging and their proposed sensing mechanism. (B) Emission spectra of the probe KSA02 after the addition of $A$. oryzae $\beta$-gal (0-1 U) and $E$. coli $\beta$-gal (0-1 U). (C) Representative images of senescent (Sct) HL-7702 cells and SKOV-3 cells incubated with KSA02; Western blot analysis of human $\beta$-gal and $\gamma \mathrm{H} 2 \mathrm{AX}$ expression levels in Sct HL-7702 and SKOV-3 cells. A schematic illustration of the distributions of SA- $\beta$-gal in Sct senescent HL-7702 cells and SKOV-3 cells is shown. Scale bar $=5 \mu \mathrm{m}$ (C, column 1 and column 2); scale bar $=10 \mu \mathrm{m}$ (C, column 3). (D) Representative images of kidney sections of mice without or with treatment with doxorubicin (DOX, 38 days), stained by KSA01 and KSA02.

Scale bar $=100 \mu \mathrm{m}$. Adapted with permission from Ref. [50]. Copyright 2021 Wiley.

Although great progress in developing fluorescent probes for the diagnosis of aging has been made, some challenges remain to be addressed. Given the high heterogeneity and the dynamics of aging in vivo, no consensus exists regarding aging biomarkers; consequently, progress has been slow in the accurate diagnosis of aging and the discovery of anti-aging drugs. Thus, developing more accurate and trustworthy strategies for aging evaluation beyond the identified age-specific biomarkers should shed light on a new paradigm for the precise diagnosis of aging. Additionally, an urgent need exists to establish an analytic platform for rapid, high-throughput screening of anti-aging drugs by using desirable small-molecule fluorescent probes. This screening platform could decrease the time and costs for discovering novel anti-aging drugs, and promote the development of anti-aging research. Ideally, in the future, new fluorescent probes will enable more accurate, sensitive and efficient diagnosis of aging, and significantly contribute to improving the human healthspan. 
A
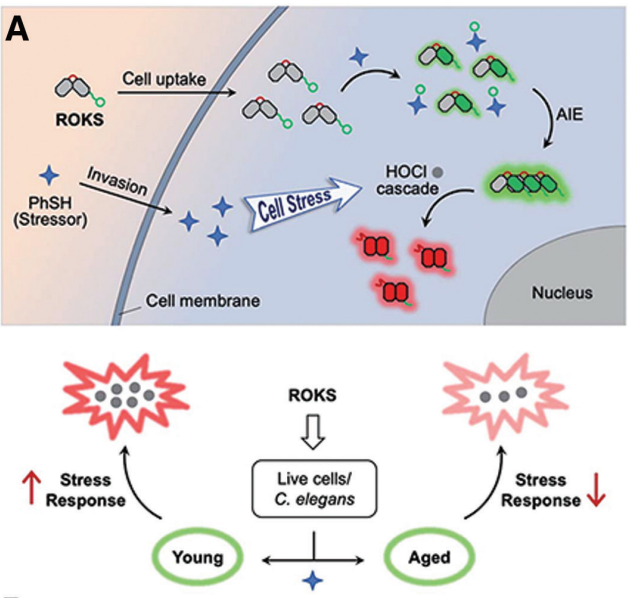

B $\mathrm{HOCl}$ site
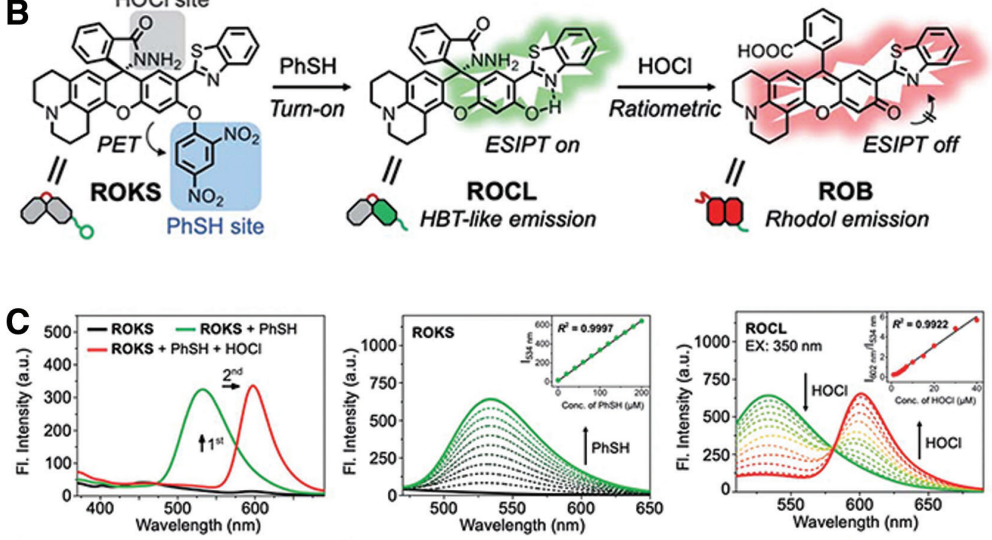

D
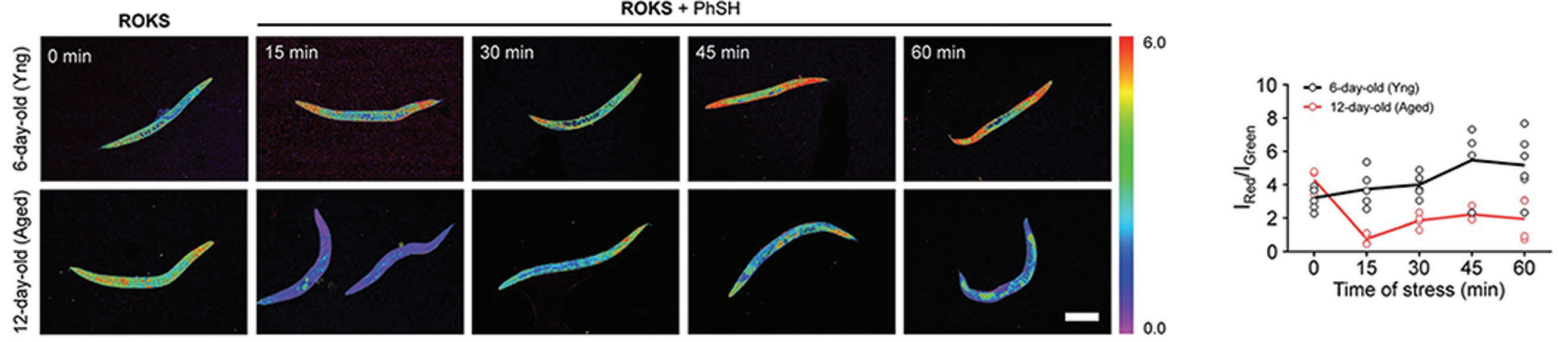

Figure 21 | (A) Schematic diagram of the dynamic evaluation of aging by using ROKS. (B) The sensing mechanism and reaction scheme of ROKS for logical detection of PhSH and HOCl. (C) Emission spectra of ROKS, ROKS after the addition of PhSH or the mixture of PhSH and $\mathrm{HOCl}$; emission spectra of ROKS after addition of PhSH; emission spectra of ROCL after the addition of HOCl. (D) Representative ratio images of C. elegans of different ages ( 6 days old and 12 days old). Young and aged C. elegans were incubated with ROKS and then treated with PhSH for various times (0-60 min). Adapted with permission from Ref. (51). Copyright 2021 The Author(s).

\section{ACKNOWLEDGEMENTS}

This work was financially supported by the National Natural Science Foundation of China (22037002, 22007032 and 21977082), the Natural Science Basic Research Plan for Distinguished Young Researchers in Shaanxi Province of China (2020JC-38), the Innovation Program of Shanghai Municipal Education Commission (2021-01-07-00-02-E00104), the Innovative Research Team of High-level Local Universities in Shanghai and the Chinese Special Fund for State Key Laboratory of Bioreactor Engineering (2060204).

\section{REFERENCES}

[1] López-Otín C, Blasco MA, Partridge L, Serrano M, Kroemer G: The Hallmarks of Aging. Cell 2013, 153:1194-1217.

[2] Niccoli T, Partridge L: Ageing as a Risk Factor for Disease. Current Biology 2012, 22:R741-R752.

[3] Campisi J, Kapahi P, Lithgow GJ, MelovS, Newman JC, Verdin E: From Discoveries in Ageing Research to Therapeutics for Healthy Ageing. Nature 2019, 571:183-192.

[4] de Magalhães JP: Longevity Pharmacology Comes of Age. Drug Discovery Today 2021, 26:1559-1562.

[5] Hernandez-Segura A, Nehme J, Demaria M: Hallmarks of Cellular Senescence. Trends in Cell Biology 2018, 28:436-453.

[6] Lee BY, Han JA, Im JS, Morrone A, Johung K, Goodwin EC, et al.: Senescence-Associated Beta-Galactosidase is Lysosomal Beta-Galactosidase. Aging Cell 2006, 5:187-195.
[7] Celeste A, Petersen S, Romanienko PJ, FernandezCapetillo O, Chen HT, Sedelnikova OA, et al.: Genomic Instability in Mice Lacking Histone H2AX. Science 2002, 296:922-927.

[8] Turenne GA, Paul P, Laflair L, Price BD: Activation of p53 Transcriptional Activity Requires ATM's Kinase Domain and Multiple N-Terminal Serine Residues of p53. Oncogene 2001, 20:5100-5110.

[9] Baker DJ, Wijshake T, Tchkonia T, LeBrasseur NK, Childs $B G$, van de Sluis B, et al.: Clearance of p16Ink4a-Positive Senescent Cells Delays Ageing-Associated Disorders. Nature 2011, 479:232-236.

[10] Hernandez-Segura A, de Jong TV, Melov S, Guryev V, Campisi J, Demaria M: Unmasking Transcriptional Heterogeneity in Senescent Cells. Current Biology 2017, 27:2652-2660.e2654.

[11] Sandhu C, Garbe J, Bhattacharya N, Daksis J, Pan CH, Yaswen $\mathrm{P}$, et al.: Transforming Growth Factor Beta Stabilizes p15INK4B Protein, Increases p15INK4B-cdk4 Complexes, and Inhibits Cyclin D1-cdk4 Association in Human Mammary Epithelial Cells. Molecular and Cellular Biology 1997, 17:2458-2467.

[12] Chen H, Ruiz PD, McKimpson WM, Novikov L, Kitsis RN, Gamble MJ: MacroH2A1 and ATM Play Opposing Roles in Paracrine Senescence and the Senescence-Associated Secretory Phenotype. Molecular Cell 2015, 59:719-731.

[13] Chen X, Varki A: Advances in the Biology and Chemistry of Sialic Acids. ACS Chemical Biology 2010, 5:163-176. 
[14] Zhu R, Wang S, Xue Z, Han J, Han S: Senescence-Associated Sialidase Revealed by An Activatable Fluorescence-On Labeling Probe. Chemical Communications 2018, 54:11566-11569.

[15] Arthur JR: The Glutathione Peroxidases. Cellular and Molecular Life Sciences 2000, 57:1825-1835.

[16] Kadota $Y$, Suzuki S, Ideta S, Fukinbara $Y$, Kawakami $T$ Imai H, et al.: Enhanced Metallothionein Gene Expression Induced by Mitochondrial Oxidative Stress is Reduced in Phospholipid Hydroperoxide Glutathione PeroxidaseOverexpressed Cells. European Journal of Pharmacology 2010, 626:166-170.

[17] Wang Y, Zhang L, Chen L: Glutathione PeroxidaseActivatable Two-Photon Ratiometric Fluorescent Probe for Redox Mechanism Research in Aging and Mercury Exposure Mice Models. Analytical Chemistry 2020, 92:1997-2004.

[18] De Colibus L, Li M, Binda C, Lustig A, Edmondson DE, Mattevi A: Three-Dimensional Structure of Human Monoamine Oxidase A (MAO A): Relation to the Structures of Rat MAO A and Human MAO B. Proceedings of the National Academy of Sciences of the United States of America 2005, 102:12684.

[19] Wang R, Han X, You J, Yu F, Chen L: Ratiometric NearInfrared Fluorescent Probe for Synergistic Detection of Monoamine Oxidase B and Its Contribution to Oxidative Stress in Cell and Mice Aging Models. Analytical Chemistry 2018, 90:4054-4061.

[20] Singh M, Piekorz RP: Senescence-Associated Lysosomal Alpha-L-Fucosidase (SA-Alpha-Fuc): A Sensitive and More Robust Biomarker for Cellular Senescence Beyond SA-Beta-Gal. Cell Cycle 2013, 12:1996.

[21] Hildebrand DG, Lehle S, Borst A, Haferkamp S, Essmann $F$, Schulze-Osthoff K: Alpha-Fucosidase as a Novel Convenient Biomarker for Cellular Senescence. Cell Cycle 2013, 12:1922-1927.

[22] Koo S, Won M, Li H, Kim WY, Li M, Yan C, et al.: Harnessing Alpha-L-Fucosidase for In vivo Cellular Senescence Imaging. Chemical Science 2021, 12:10054-10062.

[23] Di Micco R, Krizhanovsky V, Baker D, d'Adda di Fagagna F: Cellular Senescence in Ageing: From Mechanisms to Therapeutic Opportunities. Nature Reviews Molecular Cell Biology 2021, 22:75-95.

[24] Dimri GP, Lee X, Basile G, Acosta M, Scott G, Roskelley C, et al.: A Biomarker that Identifies Senescent Human Cells in Culture and in Aging Skin In vivo. Proceedings of the National Academy of Sciences of the United States of America 1995, 92:9363-9367.

[25] Liu J, Ma X, Cui C, Chen Z, Wang Y, Deenik PR, et al.: Noninvasive NIR Imaging of Senescence via In Situ Labeling Journal of Medicinal Chemistry 2021, 64:17969-17978.

[26] Chen JA, Guo W, Wang Z, Sun N, Pan H, Tan J, et al.: In vivo Imaging of Senescent Vascular Cells in Atherosclerotic Mice Using a beta-Galactosidase-Activatable Nanoprobe. Analytical Chemistry 2020, 92:12613-12621.

[27] Li Z, Cheng J, Huang L, Li W, Zhao Y, Lin W: Aging Diagnostic Probe for Research on Aging and Evaluation of Anti-aging Drug Efficacy. Analytical Chemistry 2021, 93:13800-13806.

[28] Lozano-Torres B, Blandez JF, Galiana I, Lopez-Dominguez JA, Rovira M, Paez-Ribes M, et al.: A Two-Photon Probe Based on Naphthalimide-Styrene Fluorophore for the In vivo Tracking of Cellular Senescence. Analytical Chemistry 2021, 93:3052-3060.
[29] Oushiki D, Kojima H, Takahashi Y, Komatsu T, Terai T, Hanaoka K, et al.: Near-Infrared Fluorescence Probes for Enzymes Based on Binding Affinity Modulation of Squarylium Dye Scaffold. Analytical Chemistry 2012, 84:4404-4410.

[30] Gu K, Xu Y, Li H, Guo Z, Zhu S, Zhu S, et al.: Real-Time Tracking and In vivo Visualization of beta-Galactosidase Activity in Colorectal Tumor with a Ratiometric NearInfrared Fluorescent Probe. Journal of the American Chemical Society 2016, 138:5334-5340.

[31] Li X, Qiu W, Li J, Chen X, Hu Y, Gao Y, et al.: FirstGeneration Species-Selective Chemical Probes for Fluorescence Imaging of Human Senescence-Associated Beta-Galactosidase. Chemical Science 2020, 11:7292-7301.

[32] Qiu W, Li X, Shi D, Li X, Gao Y, Li J, et al.: A Rapid-Response Near-Infrared Fluorescent Probe with a Large Stokes Shift for Senescence-Associated $\beta$-Galactosidase Activity Detection and Imaging of Senescent Cells. Dyes and Pigments 2020, 182:108657.

[33] Li Z, Ren M, Wang L, Dai L, Lin W: Development of a Red-Emissive Two-Photon Fluorescent Probe for Sensitive Detection of Beta-Galactosidase In vitro and In vivo. Sensors and Actuators B-Chemical 2020, 307:127643.

[34] Gao Z, Gao H, Zheng D, Xu T, Chen Y, Liang C, et al.: $\beta$-Galactosidase Responsive AIE Fluorogene for Identification and Removal of Senescent Cancer Cells. Science China Chemistry 2020, 63:398-403.

[35] Kim E-J, Podder A, Maiti M, Lee JM, Chung B, Bhuniya $S$ : Selective Monitoring of Vascular Cell Senescence via Beta-Galactosidase Detection with a Fluorescent Chemosensor. Sensors and Actuators B-Chemical 2018, 274:194-200.

[36] Ray PD, Huang BW, Tsuji Y: Reactive Oxygen Species (ROS) Homeostasis and Redox Regulation in Cellular Signaling. Cellular Signalling 2012, 24:981-990.

[37] Lee $\mathrm{S}-\mathrm{H}$, Lee J-H, Lee $\mathrm{H}-\mathrm{Y}, \mathrm{Min} \mathrm{K}-\mathrm{J}$ : Sirtuin Signaling in Cellular Senescence and Aging. BMB Reports 2019, 52:24-34

[38] Makau JN, Kitagawa A, Kitamura K, Yamaguchi T, Mizuta S: Design and Development of an HBT-Based Ratiometric Fluorescent Probe to Monitor Stress-Induced Premature Senescence. ACS Omega 2020, 5:11299-11307.

[39] Wang WX, Jiang W-L, Mao G-J, Tan M, Fei J, Li $Y$, et al.: Monitoring the Fluctuation of Hydrogen Peroxide in Diabetes and Its Complications with a Novel Near-Infrared Fluorescent Probe. Analytical Chemistry 2021, 93:3301-3307.

[40] Song X, He N, Wang R, Xing Y, Lv C, Yu F: Real-Time Evaluation of Hydrogen Peroxide Injuries in Pulmonary Fibrosis Mice Models with a Mitochondria-Targeted NearInfrared Fluorescent Probe. ACS Sensors 2021, 6:1228-1239.

[41] Childs BG, Durik M, Baker DJ, van Deursen JM: Cellular Senescence in Aging and Age-Related Disease: From Mechanisms to Therapy. Nature Medicine 2015, 21:1424-1435.

[42] Hwang ES, Yoon G, Kang HT: A Comparative Analysis of the Cell Biology of Senescence and Aging. Cellular and Molecular Life Sciences 2009, 66:2503-2524.

[43] Williams HP, Harwood AJ: Cell Polarity and Dictyostelium Development. Current Opinion in Microbiology 2003, 6:621-627

[44] Shi D, Hu L, Li X, Liu W, Gao Y, Li X, et al.: Lysosomal Polarity Increases with Aging as Revealed by a LysosomeTargetable Near-Infrared Fluorescent Probe. Sensors Actuators B: Chemical 2020, 319:128302. 
Acta

Materia

[45] Luzio JP, Pryor PR, Bright NA: Lysosomes: Fusion and Function. Nature Reviews Molecular Cell Biology 2007, 8:622-632.

[46] Bagh MB, Peng S, Chandra G, Zhang Z, Singh SP, Pattabiraman N, et al.: Misrouting of v-ATPase Subunit V0a1 Dysregulates Lysosomal Acidification in a Neurodegenerative Lysosomal Storage Disease Model. Nature Communications 2017, 8:14612.

[47] Zhang X, Song G-J, Cao X-J, Liu J-T, Chen M-Y, Cao X-Q, et al.: A New Fluorescent pH Probe for Acidic Conditions. RSC Advances 2015, 5:89827-89832.

[48] Wang L, Han X, Qu G, Su L, Zhao B, Miao J: A pH Probe Inhibits Senescence in Mesenchymal Stem Cells. Stem Cell Research \& Therapy 2018, 9:343.
[49] Ang J, Lee Y-A, Raghothaman D, Jayaraman P, Teo KL, Khan FJ, et al.: Rapid Detection of Senescent Mesenchymal Stromal Cells by a Fluorescent Probe. Biotechnology Journal 2019, 14:e1800691.

[50] Gao Y, Hu Y, Liu Q, Li X, Li X, Kim C-Y, et al.: TwoDimensional Design Strategy to Construct Smart Fluorescent Probes for the Precise Tracking of Senescence. Angewandte Chemie International Edition in English 2021, 60:10756-10765.

[51] Tian J, Shi D, Zhang $Y$, Li X, Li X, Teng H, et al.: Stress Response Decay with Aging Visualized Using a DualChannel Logic-Based Fluorescent Probe. Chemical Science 2021, 12:13483-13491. 